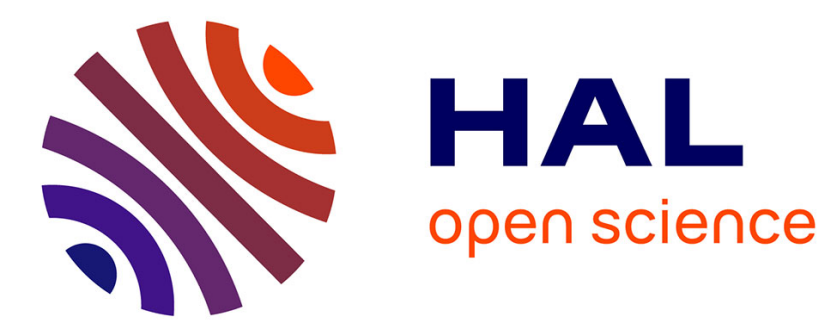

\title{
Comparing Kinetic Monte Carlo simulations with Cluster Dynamics: what can we learn about precipitation? Application to AlZr alloys.
}

Joel Lepinoux

\section{> To cite this version:}

Joel Lepinoux. Comparing Kinetic Monte Carlo simulations with Cluster Dynamics: what can we learn about precipitation? Application to AlZr alloys.. Philosophical Magazine, 2010, 90 (23), pp.3261-3280. 10.1080/14786435.2010.484403 . hal-00602608

\section{HAL Id: hal-00602608 https://hal.science/hal-00602608}

Submitted on 23 Jun 2011

HAL is a multi-disciplinary open access archive for the deposit and dissemination of scientific research documents, whether they are published or not. The documents may come from teaching and research institutions in France or abroad, or from public or private research centers.
L'archive ouverte pluridisciplinaire HAL, est destinée au dépôt et à la diffusion de documents scientifiques de niveau recherche, publiés ou non, émanant des établissements d'enseignement et de recherche français ou étrangers, des laboratoires publics ou privés. 


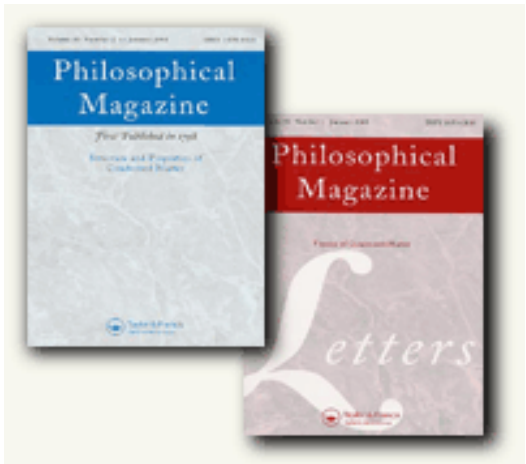

\section{Comparing Kinetic Monte Carlo simulations with Cluster Dynamics: what can we learn about precipitation? Application to AIZr alloys.}

\begin{tabular}{|r|l|}
\hline Journal: & Philosophical Magazine \& Philosophical Magazine Letters \\
\hline Manuscript ID: & TPHM-09-Nov-0484.R1 \\
\hline Journal Selection: & Philosophical Magazine \\
\hline Date Submitted by the \\
Author: & 15-Feb-2010 \\
\hline Keywords: & $\begin{array}{l}\text { Monte-Carlo, aluminium alloys, clusters, precipitation, numerical } \\
\text { modelling, numerical simulation }\end{array}$ \\
\hline Keywords (user supplied): & \\
\hline
\end{tabular}

\section{S) ScholaroNE \\ Manuscript Central}




\title{
Comparing Kinetic Monte Carlo simulations with Cluster Dynamics: what can we learn about precipitation? Application to AIZr alloys.
}

\author{
J. LEPINOUX \\ SIMAP (UMR 5266 CNRS), INPG \\ 38042 St Martin d'Hères, France
}

\begin{abstract}
Kinetic Monte Carlo simulations of precipitation in AlZr alloys are compared with predictions of Cluster Dynamics based on an enhanced thermodynamic model. A methodology and various tools are proposed to learn as much as possible from such comparisons. Important deviations between the two methods are investigated and interpreted through the role of different mechanisms.
\end{abstract}

Keywords: Cluster Dynamics, Precipitation, Nucleation, Free Energy, Coagulation.

\section{INTRODUCTION}

The goal of the present paper is multi fold:

- To propose a methodology to compare a model, mesoscopic or macroscopic, designed to predict precipitation, with Kinetic Monte Carlo (KMC) simulations,

- To apply this methodology to a particular model, i.e. Cluster Dynamics (CD), in the case study of AlZr alloys,

- To show what can be learnt about precipitation, from the combination of these two methods and additional tools, even in situations considered to be trivial.

Among classical approaches, $\mathrm{CD}$ is certainly the most rigorous way to model precipitation in binary alloys, especially when monomers are known to be the only mobile clusters. Indeed, this simple method is able to treat the different stages of precipitation, from nucleation to coalescence while avoiding usual approximations of macroscopic models (Clouet et al., 2005). The interest of modelling precipitation in binary alloys is nowadays mostly to check the validity of ingredients whose macroscopic models are built on. And comparing respective results of CD and KMC is a privileged way to improve our understanding of precipitation fundamentals. Such improvements seem highly desirable to avoid misinterpretations while trying to model much more complex situations like those encountered in modern industrial alloys.

The CD scheme is based on pairs of condensation and evaporation coefficients, noted $\beta_{n}$ and $\alpha_{n+1}$. A recently enhanced thermodynamic solution has been shown to correctly reproduce socalled equilibrium distributions of clusters in a wide range of (normalized) temperature or solubility limit in the case of $\mathrm{L}_{2}$ clusters embedded in a FCC matrix (Lépinoux, 2006 and 2009). Three situations, i.e. sets of solute concentration and temperature, concerning low solubility limit AlZr alloys were selected: $1 \%$ at $873 \mathrm{~K}, 1 \%$ at $723 \mathrm{~K}$ and $3 \%$ at $873 \mathrm{~K}$, referred to hereafter as case 1,2 and 3 , respectively. It has been shown that in these alloys only monomers are mobile, or diffuse Clouet (2004), Clouet et al. (2004). In addition, these solute concentrations are smaller than the percolation threshold of the cubic structure (1/4 of about 30\%, see Stauffer and Aharony, 1992). Thus, such alloys exhibit interesting features to test the ability of $\mathrm{CD}$ to predict precipitation 
kinetics beyond usual situations of high dilution.

Basic equations are briefly recalled in section 2 to be applied to supersaturated alloys. Sections 3 and 4 present the difficulties of comparing such a model with KMC simulations due to the small finite volume considered in atomistic simulations while $\mathrm{CD}$ is applied to a pseudo-infinite continuum. To minimize these difficulties, it is proposed to adapt the classical scheme to a finite volume. Various discrepancies are observed between $\mathrm{CD}$ and $\mathrm{KMC}$, depending on temperature and materials properties. To understand the origin of these divergences, a comparison procedure has been developed and some guidelines to get the best from such comparisons are provided. Section 5 shows that the morphological analysis of clusters built-in during KMC simulations provides new insights about precipitation. Finally, in section 6, gathering all results, some ingredients missing in $\mathrm{CD}$ to correctly reproduce $\mathrm{KMC}$ results are identified and discussed.

All KMC data mentioned in this paper were provided by courtesy from E. Clouet or obtained with the KMC code from SRMP (CEA, Saclay); details about the KMC simulations can be found in Clouet (2004) and Clouet et al. (2004).

\section{FUNDAMENTAL EQUATIONS OF CluSTER DYNAMiCS}

The fundamental equations of $\mathrm{CD}$ are briefly recalled; details about the derivation of following equations can be found in Clouet et al. (2005) and Lépinoux (2009). The structure of the master equation for the evolution of clusters of the atomic concentration $C_{n}$ of clusters of size $n>1$ writes:

$$
\dot{C}_{n}=\alpha_{n+1} C_{n+1}+\beta_{n-1} C_{n-1} \square \alpha_{n}+\beta_{n} C_{n}
$$

The evolution of monomers concentration is simply given by the conservative condition:

$$
\sum_{n=1} n C_{n}=C_{0}
$$

where $C_{0}$ is the solute concentration in the system. The pair of coefficients $\beta_{n}$ and $\alpha_{n+1}$ are the global exchange rates from class $n$ to class $n+1$ and reversely, associated with the mechanisms of monomer condensation and evaporation, respectively. These two coefficients are coupled by the relation:

$$
\frac{\beta_{n}}{\alpha_{n+1}}=C_{1} \exp \square \Delta F_{n+1}-\Delta F_{n} \not k_{B} T[
$$

where $C_{l}$ is the monomer concentration, $k_{B}$ is the Boltzmann constant, $\Delta F_{n}$ is the difference of chemical potential between that of a $n$-mer and that of $n$ monomers. The chemical potential of $n$ mers, $F_{n}$, can be written as (Lépinoux, 2009):

$$
F_{n}=F_{n}^{o}+k_{B} T \sum_{k} \ln 1-C_{k} \nabla_{k, n}
$$

It is composed of two contributions: the first one, $F_{n}^{0}$ is the free energy of (isolated) clusters assumed in thermodynamic equilibrium, while the second term is an entropy contribution of the cluster gas; in particular it accounts for the effect of frustration of the matrix ( $V_{k, n}$ is the number of atomic sites forbidden to a $k$-mer by an $n$-mer). The connection with physical time is given by the coefficient $\beta_{n}$ : 
where $D$ is the diffusion coefficient of monomers (impurity diffusion coefficient), $\omega$ is the atomic volume, $S_{n}$ and $R_{n}$ are the surface and radius of clusters, respectively.

As shown recently in Lépinoux (2009) the master equations can be rewritten in a different way to make clearer the weak coupling between thermodynamics and kinetics. Defining $\rho_{\mathrm{n}}$ as $\rho_{\mathrm{n}}=\beta_{\mathrm{n}} / C_{l}$ and $\varphi_{\mathrm{n}}$ as $\varphi_{\mathrm{n}}=\beta_{\mathrm{n}} / \alpha_{\mathrm{n}+1}$ the new form of the system is:

$$
\begin{aligned}
& \frac{\partial C_{n}}{\partial C_{1}}=\left[\square_{n+1} \rho_{n+1} C_{n+1}-\rho_{n} C_{n} \boxminus \square_{n} \rho_{n} C_{n}-\rho_{n-1} C_{n-1}\right] /\left[\frac{\dot{C}_{1}}{C_{1}}\right] \\
& \frac{\dot{C}}{C}=-\sum_{n>1} n\left[\square_{n+1} \rho_{n+1} C_{n+1}-\rho_{n} C_{n} \square \square \square_{n} \rho_{n} C_{n}-\rho_{n-1} C_{n-1}\right]
\end{aligned}
$$

Indeed, according to equation (5) $\rho_{\mathrm{n}}$ is proportional to $n^{1 / 3}$ which is a weak coupling with $n$, the cluster size. In the complete absence of coupling between $\rho_{\mathrm{n}}$ and $n$ these two equations would be simpler and cluster distributions would depend only on the coefficients $\varphi_{\mathrm{n}}$, which are purely thermodynamic quantities. Despite a coupling in $n^{1 / 3}$, equations (6-7) strongly suggest to examine the evolution of precipitation kinetics versus $C_{1}$ instead of versus the physical time, to first focus on thermodynamics and decide whether the predicted thermodynamic states are correct or not.

\section{COMPARISON OF CLUSTER DISTRIBUTIONS - MICROSCOPIC SCALE}

As in under-saturated or weakly super-saturated alloys studied in Lépinoux (2009), the key quantity to examine is the distribution of clusters, i.e. their atomic concentration versus their size, defined as the number of solute atoms they contain. Indeed, this is important information characterizing the thermodynamic state of the alloy at a given time, or equivalently at a given concentration of monomers; it will be shown later that this information is not sufficient to fully describe the system, nevertheless it remains the best starting point. The usual "precipitation kinetics" is just the evolution of these distributions with time.

\section{figure 1}

The main drawback of such a direct comparison between KMC and CD cluster distributions is that CD considers a pseudo-infinite medium while for obvious computing limitations KMC simulations necessarily deal with very small volumes. For instance, a volume of $10^{7}$ atomic sites induces a cutoff in the possible atomistic concentrations at $10^{-7}$ (cf. figure 1). Due to present computer limitations, it is not possible to investigate over a range of system sizes large enough to conclude about the absence of influence of this size constraint on cluster distributions, nevertheless this seems to be an acceptable influence. Of course, it is easy to impose similar conditions to CD: considering the same volume of material than in KMC simulations, classical CD equations are applied to integers (i.e. number of clusters) instead of floating numbers (i.e. concentration of clusters). When appropriated, some randomness is introduced following classical algorithms to solve properly numerical operations with integers, which does not modify results of CD calculations, disregarding normal statistical variations. The main advantage of this adaptation of CD is that the lengths of respective cluster distributions can now be compared. Unfortunately, the direct 
comparison of such cluster distributions is even more difficult to interpret due to the increasing number of empty classes of size, now in both distributions. To by-pass this difficulty, instead of comparing the direct cluster distributions, it is highly preferable to consider the cumulative distributions, defined as follows:

$$
C_{n}=\sum_{k=1}^{n} k C_{k}
$$

Compared with direct distributions, an advantage of these cumulative distributions is to exhibit a much lower dispersion over various runs and a low dependence on the size of the simulation box, provided this one is larger than a certain size, depending slightly on the imposed conditions (for the present problem, $10^{6}$ atomic sites is an very acceptable minimum). In principle, one could fit these curves with continuous functions and compare their first derivative, i.e. to rebuild equivalent direct distributions; but this procedure is rather cumbersome and can be source of various errors. Analysing these cumulative distributions is less trivial but much safer. Before any particular comparison some general and simple properties can be guessed about these cumulative distributions. Most of divergences should concern classes of large size and as the concentrations of these classes are very small compared with the monomer concentration, these deviations are likely to be stable. On the contrary, the deviations concerning classes of small size should vanish much faster due to the strong tendency of the system to come back at equilibrium whatever the origin of deviations. Of course, comparisons between the two methods have to be done for the same concentration of monomers, as done in figures 1 and 2. Now we consider the respective shapes of cluster distributions obtained with $\mathrm{KMC}$ and $\mathrm{CD}$, as well as their evolution.

- Case 1: $C_{0}=1 \%, T=873 \mathrm{~K}$

At the beginning, until precipitation starts, with a critical size $n^{*}=19$, both distributions are in perfect agreement. After the beginning of precipitation a small divergence appears, larger for large classes as expected. This divergence propagates towards classes of small size then decreases as a plateau appears within the cluster cumulative distribution. Such a plateau corresponds to a widening hole within the direct distribution of cluster size, due to the increasing separation between supercritical clusters and the solid solution. Of course, at a given point of this evolution, the width of this no-cluster range might slightly depend on the size of the system but it is not an artefact. Finally only a small residual translation persists in the last part of the curve while the critical size is around 30 (beware of the logarithmic scale in abscissa). In first approximation, the observed differences are small enough to conclude that for weakly supersaturated alloys CD reproduces fairly well KMC distributions and in this case, it is not necessary to improve the $\mathrm{CD}$ algorithm. However the observed deviations are interesting to understand, in relation with the two following cases, of higher supersaturation.

- Case 2: $C_{0}=1 \%, T=723 \mathrm{~K}$

As in case 1 , before precipitation starts with a critical size $n^{*}=7, \mathrm{KMC}$ and $\mathrm{CD}$ distributions are in excellent agreement. Then, note that deviation which develops has the opposite sign to that observed in case 1. In addition the amplitude of this deviation is much larger and concerns a much larger fraction of classes of cluster size. In this case, this means that the growth of clusters is faster than expected. A possible mechanism will be discussed later.

\section{- Case 3: $C_{0}=3 \%, T=873 \mathrm{~K}$}

In this case a new type of deviation appears at the very beginning and includes all classes except monomers, by construction. This deviation, of same sign as in case 2, corresponds with the triggering of precipitation with a critical size $n^{*}=8$, much earlier than expected according CD. Then this deviation increases in amplitude up to a maximum and, as in other cases, distributions converge. Then a new divergence develops starting from classes of large size as observed in the 
previous case.

\section{figure 2}

At this stage we have identified three different possible scenarii of deviation for KMC cluster distributions versus those predicted by $\mathrm{CD}$. One could examine the evolution of the monomer concentration versus time; of course, other deviations are observed. As they are not helpful to understand why thermodynamic states are different, they are not reported here. Thus, to understand what these deviations mean, we need to get more information about the clusters. A simple and classical way is to report the evolution of average quantities characterizing cluster distributions as proposed in the next section.

\section{COMPARISON OF CLUSTER DISTRIBUTIONS: MACROSCOPIC SCALE}

Indeed, although in KMC simulations the location of every solute atom is available at any time, for the sake of simplicity, the classical outputs are average quantities which can be obtained from the analysis of cluster distributions. For this purpose, it is usual to take the critical size $n^{*}$ of clusters as defined by the classical nucleation theory (Clouet et al., 2005) as the threshold to distinguish stable precipitates from unstable clusters, during the whole kinetics. Thus, at any time, only clusters of size $n$ larger that this theoretical critical size are counted as precipitates and the provided outputs are typically:

- the atomic concentration of precipitates $N_{p}$

- the average size of precipitates $\bar{n}$

- the atomic fraction of precipitates $F_{p}=N_{p}{ }^{n}$

By construction, only two of these three quantities are independent, thus one of them can be disregarded. If one wants to compare with experimental data, it is obvious that the two first quantities are the most convenient (see for instance (Clouet et al., 2005)). But to compare numerical results the average size of clusters should be avoided; indeed, the usual shape of obtained curves is highly inappropriate for an in-depth comparison, contrary to that reporting the evolution of $F_{p}$ which exhibits a more regular slope. Thus, here, only $F_{p}$ and $N_{p}$ will be used to compare CD and KMC precipitation kinetics, while the average size will be disregarded. Figure 3a shows the usual representation mode of these quantities, i.e. their evolution versus the physical time, while figure $3 \mathrm{~b}$ shows the evolution of the same quantities versus $\left(1-C_{1}\right) / C_{0}$, both for case 1 . For CD results, two solutions are compared:

- CD 1) the classical solution, with a constant free surface energy, here taken from Clouet et al., (2004)

- CD 2) the complete solution, i.e. equation (4)

figure 3

- Case 1: $C_{0}=1 \%, T=873 \mathrm{~K}, n^{*}=19$ (figure $3 a-b$ )

If one considers only figure $3 \mathrm{a}$ there is a clear advantage to the second solution for the prediction of the number of precipitates but for the precipitated fraction it is difficult to conclude. Now if one considers figure $3 \mathrm{~b}$, there is no more ambiguity: the second solution is almost correct, the beginning is perfect, then the predicted concentration of precipitates is $20 \%$ too small but later the two curves converge, confirming figure $2 \mathrm{a}$. On the contrary, the first one is definitely disqualified. As explained in Lépinoux (2009) thermodynamics and kinetics cannot be completely separated; nevertheless, to understand the deviations of cluster distributions reported in the previous section, the representation mode adopted in figure $3 \mathrm{~b}$ is the most efficient one to test various approximate solutions. Consequently, only this representation mode will be used for the two other cases to focus 
on the thermodynamic aspects.

- Case 1: $C_{0}=1 \%, T=723 \mathrm{~K}, n^{*}=7$ (figure $3 c$ )

Again, the precipitated fraction predicted by $\mathrm{CD}$ is almost perfect, only a tiny difference can be noted at the very beginning. The concentration of precipitates exhibits the same kind of deviation as the precipitated fraction at the very beginning of precipitation, then it is correct up to the maximum of this curve. After the peak, a noticeable deviation appears and there is no evidence of convergence. Again this confirms the increasing deviation observed in figure $2 \mathrm{~b}$.

- Case 3: $C_{0}=3 \%, T=873 \mathrm{~K}, n^{*}=8$ (figure $3 d$ )

The most streaking feature is that the CD solution clearly fails to reproduce the beginning of the precipitation kinetics although it converges and is in fairly good agreement up to the $N_{p}$ peak. However a deviation similar to that observed in case 2 seems to develop after this peak even if it is at a lesser extent. Note also that the deviation observed at the beginning of the kinetics is of the same type than in the case 2, it is just much more pronounced. This suggests that the early deviation observed in figure $2 \mathrm{c}$ has a short time-life in case 2 and has been overlooked.

Finally, it seems that case 2 and 3 exhibit a main type of deviation but also a secondary one, the roles being inversed between these two cases. The information provided by such comparisons is rather poor but at least it confirms the existence of major discrepancies between KMC and CD results in a more conventional way.

$\mathrm{CD}$ is based on two strong hypotheses worth examining:

- clusters are at thermodynamic equilibrium

- diffusion of monomers is the only mechanism for growth or decay of clusters

\section{Characterizing THE MORPHOLOGY OF KMC ClUSTERS}

A great advantage of $\mathrm{KMC}$ is that coordinates of the atoms are available at any time. Thus at a given time, or concentration of monomers, it is possible to visit all clusters and to compare their morphology with the predicted one for clusters at thermodynamic equilibrium. For that purpose, we need to define some properties, necessarily related to the free energy of clusters. It is recalled that the free energy term $F_{n}^{0}$ for clusters at thermodynamic equilibrium can be calculated by a MonteCarlo technique based on the sampling of two quantities, namely the capture and the release rate, defined as follows, respectively (Lépinoux, 2005 and 2009):

$$
\begin{aligned}
& P_{n, n+1}^{0}=\sum N_{b o n d s}^{\text {out }} \exp \square N_{\text {bonds }}^{\text {in }} T_{w} / T \mathbb{q}_{n} \\
& P_{n+1, \mathrm{n}}^{0}=\sum N_{\text {bonds }}^{\text {out }} \exp -N_{\text {bonds }}^{\text {in }} T_{w} / T \mathbb{\square}_{n+1} \square
\end{aligned}
$$

Subscripts $(n)$ and $(n+1)$ mean that the thermal averages are calculated over ensembles of configurations of size $(n)$ and $(n+1)$ respectively. $N_{b o n d s}^{\text {in }}$ and $N_{b o n d s}^{\text {out }}$ are the number of bonds between the site where a solute atom is going to be exchanged with a solvent atom, with the cluster ("in") or with the next shell ("out"), respectively.

The ratio of these two quantities is related to the difference of free energy between configurations of $(n+1)$ solute atoms in one hand and that of a configuration of $n$ solute atoms and a monomer on the other hand, as follows:

$$
\frac{P_{n, n+1}^{0}}{P_{n+1, n}^{0}}=\operatorname{Exp}-\frac{F_{n+1}^{0}-F_{n}^{0}-F_{1}^{0}}{k T}[
$$


However, the relevance of these coefficients and their ratio is not restricted to the calculation of $F_{n}^{0}$. Indeed, they characterize the morphology of the cluster-matrix interface; thus they can be used to reveal and quantify a deviation of the cluster morphology versus their average shape at thermodynamic equilibrium (Lépinoux, 2009). Multiplying the capture factor by $C_{1}$ allows to put into evidence the local critical cluster size $n^{*}$ defined by the value of $n$ such that $C_{1} P_{n, n+1}^{0}=P_{n, n-1}^{0}$. This local critical size coincides with the usual macroscopic one only in highly dilute alloys where the second term in equation (4) is negligible.

Whatever the total solute concentration, the monomer concentration and the temperature, these two as-defined quantities exhibit a clear change of behaviour around $n^{*}$ where they respective curves intersect and define two regions as shown in figure 4 for case 1:

$-n<n^{*}$, disregarding statistical fluctuations, the predicted values (equilibrium) superimpose pretty well with the KMC data, as expected.

- $n>n^{*}$, the KMC data no longer behave as expected. Both factors increase much faster than expected: this is the signature of cluster morphologies more extended, in average, than the average one at equilibrium.

The release factor characterizes the cluster side of the cluster-matrix interface and is consequently more sensitive to details of the cluster morphology than the capture factor, related to the matrix side. This explains why the deviation of the release factor is always larger than that of the capture factor. Then the question to address is whether it is a general rule or particular to weakly supersaturated alloys as in case 1 and at some steps of the precipitation kinetics.

In figure 5, the evolution of the release factor was plotted for increasing values of $n^{*}$, while $C_{1}$ decreases. As can be seen, the deviation continues to develop while $n^{*}$ increases, and the larger the size of precipitates, the larger the deviation of their morphology from the equilibrium shape, in all cases. This confirms that the relative weight of extended configurations is higher than at thermodynamic equilibrium, whatever the supersaturation. In the two first cases, i.e. with $C_{0}=1 \%$, the starting of the deviation corresponds pretty well with the value of $n^{*}$. But in case 3, i.e. with $C_{0}=3 \%$, note that this seems true only at the very beginning of precipitation; then the deviation tends to start earlier and earlier; for the last curve it starts for $n$ about half of $n^{*}$. Note that for a given cluster size, in the first approximation the deviation is quite the same for the various distributions, for a given case. Nevertheless this deviation seems to slowly decrease. This is particularly clear in figure $5 \mathrm{~b}$ for the last set of KMC values. Among all analysis provided here, this is the best clue of convergence toward equilibrium in the late stage of precipitation (of the investigated range). Note also for $n<n^{*}$ a deviation of opposite sign clearly appears. All these points will be discussed later.

Thus, the examination of coefficients $P_{n, n+1}^{0}$ and especially $P_{n+1, \mathrm{n}}^{0}$ provides interesting information about the change of morphology of clusters as a function of their size during precipitation, nevertheless, according $\mathrm{CD}$, it is their ratio, $P_{n, n+1}^{0} / P_{n+1, \mathrm{n}}^{0}$ (equation 11), which is the relevant quantity from a thermodynamic point of view. Strictly speaking, this quantity cannot be extracted from KMC results, nevertheless the quantity $P_{n, n+1}^{0} / P_{n, n-1}^{0}$ is an excellent approximation provided that $n$ is greater than typically 10. To improve the accuracy of comparisons, the KMC data were compared with expected values of $P_{n, n+1}^{0} / P_{n, n-1}^{0}$, instead of the ratio $P_{n, n+1}^{0} / P_{n+1, \mathrm{n}}^{0}$; again the physical information is quite the same. The results reported in figure 6 for increasing values of $n^{*}$, as in figure 5, are more or less dispersed due to the limited size of the system but all exhibit the same global trend. Up to $n^{*}$ (approximately), again clusters exhibit the same properties as at thermodynamic equilibrium, while for larger values the ratio deviates more and more toward lower 
values, due to the deviation of $P_{n+1, \mathrm{n}}^{0}$ higher than that of $P_{n, n+1}^{0}$. Apart this general behaviour the particularities observed in figure 5 for cases 2 and 3 can be observed here too, but at a lesser extent, due to the less sensitive $P_{n, n+1}^{0}$ coefficient. Nevertheless this seems to confirm some change in cluster morphology, some kind of "recovery" in the late step, especially in figure 6b. Note in figure $6 c$ that the properties of clusters seem to be fairly independent of their size, at least in the final distributions. This point will be also discussed later.

\section{Dispersion}

One of the last sets of data $\left(n^{*}=19\right)$ in figure $6 \mathrm{c}$ is analysed again in figures 7 and 8 to focus on two aspects. As indicated in the figure captions, the points plotted in previous figures are averages for given cluster sizes, which hides the dispersion per class of size. Figure 7 shows that this dispersion is particularly large in the middle of the distribution. The reason is that only one value is possible for the 2-mers, i.e. at the beginning of the curve, then the range of possible values increases very quickly and classes are still reasonably populated to explore this range, while at the end of the distribution, classes are represented only by one or a few clusters, when not empty, thus the range of dispersion decreases quickly after a maximum. This dispersion is a feature usually disregarded, which is fully justified for approaches historically dedicated to low temperatures and high dilution situations. Nevertheless, it is clear that improving modelling of precipitation should also consider this aspect.

\section{Macroscopic and microscopic critical sizes}

Through the same example as in figure 7, figure 8 illustrates the confusing concept of critical size. First we compare the ratio $P_{n, n+1}^{0} / P_{n, n-1}^{0}$, for a given value of $C_{l}$, with its expected values at equilibrium, as done in figure 6 . On the same figure we report also the ratio $\beta_{n} / \alpha_{n} C_{1}[$. The classical critical size $n^{*}$ is defined by the condition $\beta_{n}=\alpha_{n}$ in any case, but, in the limit of infinite dilution, this condition is equivalent to $C_{1} P_{n, n+1}^{0}=P_{n, n-1}^{0}$ because the second term in equation (5) is negligible in this case. A simple way to compare the conditions of criticability according these two criteria is indicated in figure 8 by a unique horizontal line of height $1 / C_{1}$ (normalized by the same factor as other quantities) which defines the sub- and super-critical ranges. Of course this defines two distinct critical sizes which here differ by a factor 2, approximately, which is still a small difference compared with alloys of high solubility limit (see Lépinoux (2009) for an example). Again, the examination of the first quantity shows that in the subcritical region, in average, clusters are in equilibrium, while in the supercritical range, they deviate so much that most classes remain close to or even under the stability threshold. Now, coming back to figure 7 , it can be seen that not all clusters of size $n>n *$ are supercritical or similarly not all clusters of size $n<n *$ are subcritical, their state depending first of all on their morphology, not on their size. Now if one examines the second quantity, in average all classes of size larger than the macroscopic critical size are clearly super-critical. This value of macroscopic critical size compares well with the limit of agreement of $\mathrm{P}_{\text {release }}$ with the equilibrium one already mentioned about figure $6 \mathrm{c}$.

Thus to summarize, the behaviour of $\mathrm{P}_{\text {release }}$ is in good agreement with that of the macroscopic critical size while the behaviour of $\mathrm{P}_{\text {capture }} / \mathrm{P}_{\text {release }}$ is in good agreement with the local critical size. A tentative explanation is proposed:

- $P_{\text {release, }}$ as already mentioned is among other features one possible way to finely characterize the structure of clusters surface; it is very sensitive to their morphology. The agreement between the change of behaviour of $P_{\text {release }}$ around the macroscopic critical size suggests that the structure of clusters interfaces reflect the growth and decay mechanisms, associated to the definition of the macroscopic cluster size. Whatever these mechanisms it sounds consistent.

- Like $\mathrm{P}_{\text {release }}, \mathrm{P}_{\text {capture }}$ contains information about the first layer around clusters, the one where exchange takes place, but also on the next one in the matrix which is assumed empty. For the purpose of calculating the free energy of isolated clusters this is absolutely correct. And by 
Deviations of cluster morphology from equilibrium influence the properties of clusters and then the global precipitation kinetics. If they are more compact than at equilibrium, they are more stable, therefore they better resist to dissolution. Therefore, when dissolution prevails, poorly connected solute atoms are first released and the shape of clusters is slowly driven toward compact configurations, which decreases the release rate. In contrast, when coarsening prevails, solute atoms or local fluctuations are captured mostly at random, driving the cluster shapes toward less stable configurations, which, this time, decrease the coarsening rate. Thus, although the reasons are opposite, the two effects tend to slow down the precipitation kinetics.

\section{COAGULATION}

Obviously the morphological deviations of clusters should influence the precipitation kinetics, but one can wonder if it is an important contribution to the observed discrepancies between KMC simulations and CD. The interest of a simple framework like CD is that it is easy to test almost any assumption. Thanks to data reported in the previous section it is fairly easy to mimic the deviations or the ratio $P_{n, n+1}^{0} / P_{n+1, n}^{0}$ from their equilibrium values. Surprisingly, if nothing else is changed in the $\mathrm{CD}$ scheme, morphological deviations of super-critical clusters have a minor effect on precipitation kinetics compared with their amplitude. Again this suggest that the main reason for observed differences between KMC and CD results, at least in the most supersaturated cases 2 and 3 , is related to some kinetic mechanisms which are not accounted for in CD. The observed morphological deviations, mostly of super-critical clusters, are just a consequence of these mechanisms and not the main cause of discrepancy between KMC and CD, even if of course they do participate.

Detailed observations of cluster morphology in the 3D space at various temperatures and concentrations suggests a possible mechanism which could be called "static-coagulation" by opposition to classical coagulation which assumes a non-zero mobility of clusters, depending on their size (see for instance Athènes et al. (2000), Bréchet and Martin (2006) and Mao et al. (2007), Soisson and Chu-Chun (2007)). An easy way to reveal this mechanism is to observe the growth of precipitates depending on the solute concentration.

Figure 9 shows several snapshots of the growth of an isolated cluster with conditions: $C_{0}=2.525 \%$, $T=800 \mathrm{~K}$. These conditions were chosen for computing purposes, to obtain a weakly super-saturated alloy at an intermediate temperature between that of case 2 and 3 . When the size of the cluster is about 100, fluctuations are rather large compared with cluster size but the shape remains roughly cubic. At large size, i.e. more than 10000 solute atoms, $\{100\}$ facets are still clearly visible although the global shape is more rounded. Although from time to time a large fluctuation is captured which seriously perturbs the shape of the cluster for a long time (see figure 9c), growth occurs mostly by capture of monomers and small fluctuations.

Figure 10 shows a completely different scenario at $\mathrm{T}=873 \mathrm{~K}, C_{0}=3 \%$ (10a) and $C_{0}=5 \%(10 \mathrm{~b})$. These alloys are highly super-saturated which induces many super-critical clusters in the KMC simulation box. Only clusters containing more than 100 solute atoms are shown. Contrary to the previous case, here clusters look composed of several smaller clusters connected by bridges of various strength. Although the number of clusters increases and their average size too, it is difficult to predict which 
ones will survive as sub-clusters often dissociate and re-associate with their neighbours and the complexity of clusters increases with $C_{0}$. This is fully consistent with the important deviation of super-critical clusters properties as indicated in figures 6 to 8 . The quasi constant ratio $P_{\text {capture }} / P_{\text {release }}$ in the range of size 20-60, in figure 7 for instance, can even be seen as the signature of some kind of self-similarity due to the intense activity of recombination and fragmentation of clusters preventing the growth of well-formed precipitates. Of course the existence of two sub-lattices increases this trend, impeding the merging of some close clusters. Coagulation based on mobility of small clusters is now well documented but the influence of the kind of static coagulation, requiring only monomer diffusion, has been largely neglected. In addition, it cannot be treated in a generic way, as the ability of clusters to reorganize after the capture of a fluctuation strongly depends on the details of the atomic potential for the considered alloy. In the present case, a complete reorganization is almost impossible due to strong repulsive interaction between first neighbours which prevents a vacancy from exploring a cluster in depth.

A variant of this mechanism which occurs mostly at the beginning of case 3 and at a much lesser extent in the very beginning of case 2 is what could be called "geometrically necessary coagulation". It results from the initially high concentration of monomers while "static coagulation" defined above results also from a high supersaturation. Indeed, when the solute concentration increases, the available space for diffusion of monomers decreases and early reorganization is impossible without forming clusters of size much larger than expected for that concentration of monomers. As a consequence, the concentration of small clusters is much lower than expected at equilibrium; nevertheless, after reaching a maximum of deviation, the two curves converge (see figure 2c). Figure 11 shows the proportion of frustrated sites for monomers, i.e. the proportion of atomic sites where a monomer cannot be located without bonding with other solute atom. This ratio strongly depends on the solute concentration. When $C_{0}=1 \%$, whatever the temperature, when reported versus $\left(1-C_{l} / C_{0}\right)$ as previously, before slowly decreasing, this ratio exhibits a plateau. The beginning of precipitation already corresponds to the decreasing part of these curves (compare with figure 3). But for case 3, i.e. with $C_{0}=3 \%$, instead of a plateau one observes a clearly increasing and rather long step, whose maximum around $C_{l} / C_{0}=0.4$ corresponds well with the maximum of discrepancy between KMC and CD cluster distributions (see Figure 2c). After that, the two curves converge and the fraction of frustrated sites quickly decreases. Such behaviour suggests that some rearrangements during the incubation stage are necessary then thermodynamics plays its normal role. Generally speaking, coagulation is a perturbation of the fundamental mechanism which remains the exchange of monomers. The late stage in case 2 is another illustration of this principle: when the supersaturation is low enough, clusters slowly recover their equilibrium properties.

As space is not a direct ingredient of the CD scheme, in its present state this method is not able to deal with the finite nature of the number of atomic sites. Thus, such an effect cannot be accounted for without serious modifications.

Of course these coagulation mechanisms are not very difficult to mimic in an empirical modified $\mathrm{CD}$ able to fit any KMC kinetics. However nothing worth noting could be learnt from such an empirical approach. Extending CD as a predictive tool for concentrated alloys requires such effects to be modelled in a self-consistent way.

\section{CONCLUSION}

A detailed procedure to compare KMC simulations with predictions of models treating precipitation at a meso or macro scale has been proposed and applied to CD. The first step consists of reporting global quantities like the concentration of precipitates and the precipitated fraction versus $\left(1-\mathrm{C}_{1} / \mathrm{C}_{0}\right)$ instead of versus the physical time. The advantage of this representation is to focus on 
thermodynamic properties to better detect possible discrepancies between the two methods and avoid some trivial misinterpretations. Indeed, as long as the thermodynamic states are not equivalent and their differences not understood, discussing their evolution versus time is premature. The second step consists of examining the evolution of cumulative cluster concentrations that are the fundamental signature of precipitation kinetics. Finally the third step is based on the analysis of cluster morphology, applying a technique already used to calculate the free energy of clusters at thermodynamic equilibrium. Direct observations of 3D configurations allow these analyses to be confirmed.

Two fundamental assumptions have been investigated in detail with the help of these various tools. It has been shown that in the first approximation the assumption that clusters are at thermodynamic equilibrium is correct in the sub-critical range, but super-critical clusters exhibit noticeable deviations of morphology and then of properties. In this context, "thermodynamical equilibrium"means that clusters of a given size exhibit the same properties, in average, than those of an isolated cluster free to explore the whole range of its possible configurations in an infinite medium. The use of the ratio $\mathrm{P}_{\text {capture }} / \mathrm{P}_{\text {release }}$ to characterize the properties of clusters does not seem appropriate for highly supersaturated alloys. Again this is related to the existence of a competition between the usual growth mechanism based on the migration of monomers and the capture of other clusters formed at close distance. On the contrary, $\mathrm{P}_{\text {release }}$ is able to detect the deviation of cluster morphology due to these unexpected captures. Two mechanisms, referred to as "static" and "geometrically necessary" coagulations, have been put into evidence; the distinction between the two mechanisms is of course mostly a question of convenience. These mechanisms, undoubtedly enhanced by the $\mathrm{L}_{2}$ structure of clusters studied here, are likely to be observed also in segregation and are rather difficult to model in a self-consistent way.

Cluster Dynamics is definitely a remarkable tool when coupled with KMC simulations to explore the various facets of precipitation but should be used with caution to predict precipitation kinetics when the solute concentration exceeds some threshold, here of the order of $1 \%$ atomic. Accounting for all features revealed in the present work appears in contradiction with the expected simplicity of such a model. A preliminary exploration of a given problem with Kinetic Monte Carlo simulations and an analysis of the observed discrepancies following the procedure proposed here should allow to evaluate the magnitude of errors and decide whether the use of Cluster Dynamics is acceptable or not for that problem. Modelling these complex mechanisms in a self-consistent way remains a challenge and preserving the simplicity of classical CD seems impossible. Such a general scheme, based on the examination of higher solute concentration cases, will be reported later.

\section{References}

[1] CLOUET, E., BARBU, A., LAE, L. and MARTIN, G., 2005, Acta Materialia., 53, 2343

[2] LEPINOUX, J., 2006, Phil. Mag., 86, 5053.

[3] LEPINOUX, J., 2009, Acta Materialia., 57, 1086.

[4] CLOUET, E. 2004, $\mathrm{PhD}$ thesis, Ecole Centrale, Paris. Available online at: tel.csd.cnrs.fr/documents/archives0/00/00/59/74

[5] CLOUET, E., NASTAR, M. and SIGLI, C., 2004, Phys. Rev. B, 69, 064109.

[6] STAUFFER, D. and AHARONY, A., Introduction to Percolation Theory, London, Taylor and Francis, 1992.

[7] LEPINOUX, J., 2005, Philosophical Magazine, 85, 3585

[8] ATHENES, M., BELLON, P. and MARTIN, G., 2000, , Acta Materialia., 48, 2675

[9] BRECHET, Y. and MARTIN, G., C.R. Physique, 2006, 7, 959.

[10] MAO Z., SUDBRACK, C., YOON, K., MARTIN, G. and SEIDMAN, D., 2007, Nature Materials, 6, 210.

[11] SOISSON, F. and Chu-Chun Fu, 2007, Phys. Rev. B, 76, 214102 


\section{Acknowledgments}

This work greatly benefited from the joint research program "CPR Precipitation" (Arcelor, AlcanPechiney, CNRS and CEA) and GDR "Transdiff". Various discussions with Drs. G. Martin, F. Soisson, A. Finel, P. Guyot and C. Sigli are gratefully acknowledged. The author is especially grateful to Dr. E. Clouet for helpful discussions and for providing his KMC results and code.

\section{Figure captions}

Figure 1: An example of comparison of cluster distributions obtained with KMC (symbols) and CD (lines). Here the size of the KMC simulation box was $810^{6}$ atomic sites which imposes a cut-off value of $1.2510^{-7}$ to cluster concentrations. For the two first sets of distributions this cut-off value is not a major drawback but the comparison becomes meaningless for the third case due to the finite size of the KMC box. Letters A, B and E refer to distributions shown in figures 2 and 3.

Figure 2: Comparison of KMC cumulative cluster distributions (symbols) with those expected at equilibrium (plain lines) for cases 1 (a), 2 (b) and 3 (c). In (a) letters A to E refer to particular distribution shown also in figures 1 and 3.

Figure 3: Comparison of two CD solutions with KMC simulations:

- (a) and (b) $\mathrm{AlZr} 1 \%$ at $\mathrm{T}=873 \mathrm{~K}$ (case 1 )

- (c) AlZr $1 \%$ at $\mathrm{T}=723 \mathrm{~K}$ (case 2)

- (d) $\mathrm{AlZr} 3 \%$ at $\mathrm{T}=873 \mathrm{~K}$ (case 3)

The reported quantities are the precipitated fraction (plain symbols) and the number of precipitates (open symbols) multiplied by a convenient constant to fit the window. Results are reported vs. time (a) or vs. $\left(1-\mathrm{C}_{1}\right) / \mathrm{C}_{0}(\mathrm{~b}, \mathrm{c}$ and $\mathrm{d})$ where $\mathrm{C}_{1}$ is the concentration in monomers and $\mathrm{C}_{0}$ the total solute concentration.

The two CD solutions are:

CD 1, with constant free surface energy, dashed line (magenta on line)

CD 2, the complete solution, plain line (red online)

Letters $\mathrm{A}$ to $\mathrm{E}$ in (b) refer to distributions shown in figure 2 (a) and figure 1.

Figure 4: Typical behavior of the capture and release factors measured on KMC clusters for early (a) and late (b) stages of precipitation, compared with expected values at equilibrium (plain lines). The intersection of the two curves defines the instantaneous local critical cluster size $n^{*}$. NB: to simplify, both factors are normalized by $\exp \left(-T_{w} / T\right)$ so that $\mathrm{P}_{2,1}=10$, an integer whose value can be found in (Lépinoux, 2005). 
Figure 9: Typical growth of an isolated precipitate from a nucleus containing about 100 solute atoms (a) up to its final size about 15000 solute atoms (d), in equilibrium with the solid solution. The solute concentration, a few $10^{-3}$, was chosen high enough to trigger precipitation in a reasonable amount of computation time and small enough to select only one precipitate in the KMC simulation box $\left(10^{6}\right.$ atomic sites $)$ at $\mathrm{T}=873 \mathrm{~K}$. The macroscopic shape is roughly cubic with $\{100\}$ faces. Although at such low solute concentration the precipitate remains fairly compact, growth is not monotonic and important changes of shape due to the capture of nearby fluctuations of various size can be observed. (e) color chart for solute atoms as a function of the number of links with other solute atoms.

Figure 10: Typial shapes of clusters observed at solute concentration of $1 \%$ (a) to $5 \%$ (b), $\mathrm{T}=873 \mathrm{~K}$. Clusters are increasingly composed of aggregates of smaller clusters loosely interconnected. Although the size of clusters increases, the largest ones are not necessarily the most stable ones (cf. figure 9 for the color chart).

Figure 11: Evolution of the fraction of atomic sites where a monomer cannot be located without bonding with another solute atom, as a function of $\left(1-C_{1}\right) / C_{0}$ where $C_{1}$ is the concentration in monomers and $C_{0}$ the total solute concentration. For $C_{0}=1 \%$ the first stage is a plateau, while for $C_{0}=3 \%$ a bump appears before a fast decrease. 


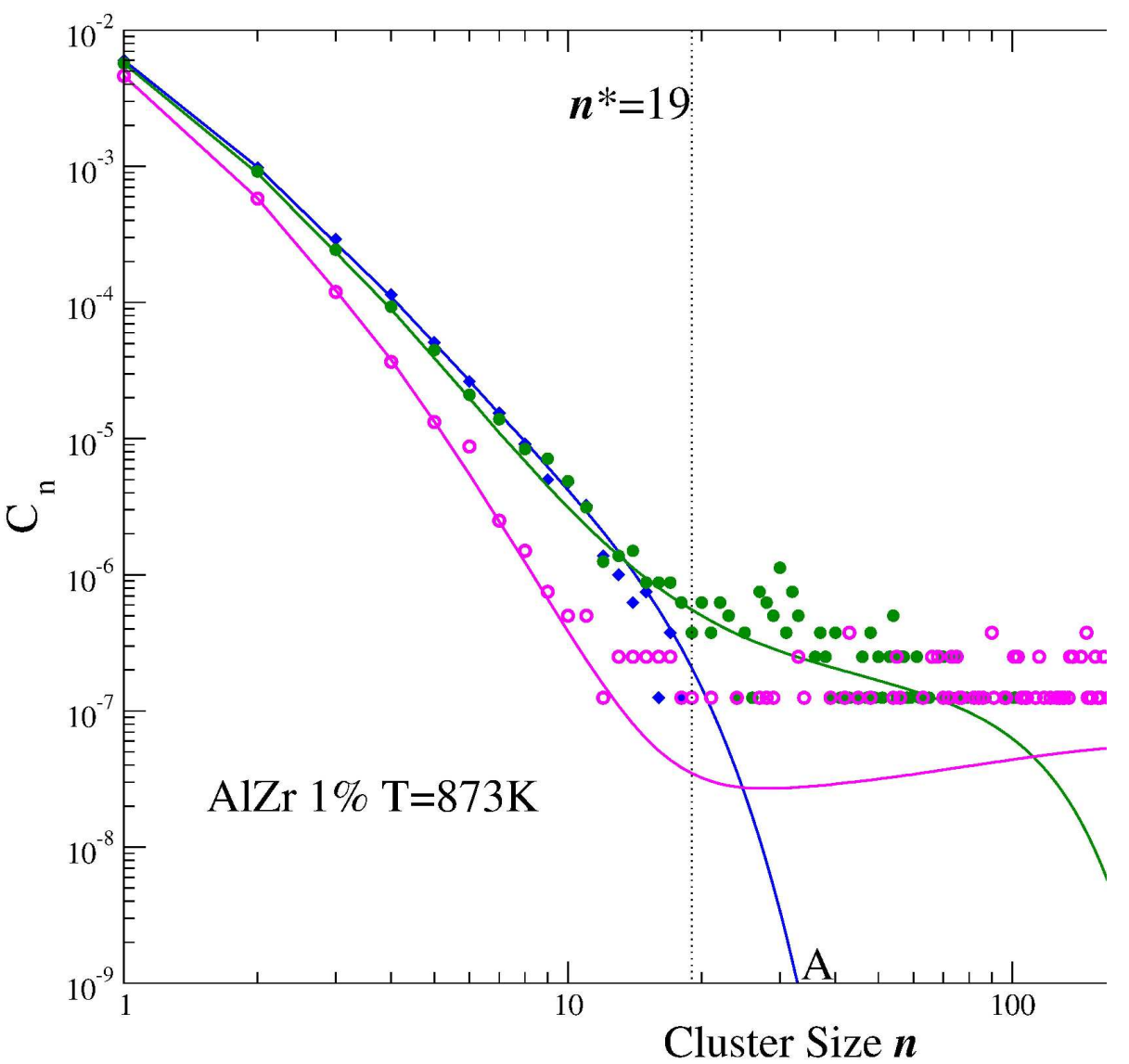

Figure 1: A typicalcomparison of cluster distributions obtained with KMC (symbols) and CD (lines). Here the size of the KMC simulation box was 8106 atomic sites which imposes a cut-off value of 1.25 10-7 to cluster concentrations. For the two first sets of distributions this cut-off value is not a major drawback but the comparison becomes meaningless for the the third case due to the finite size of the KMC box. Letters A, B and $E$ refer to distributions shown in figures 2 and 3.

$215 \times 279 \mathrm{~mm}(600 \times 600 \mathrm{DPI})$ 


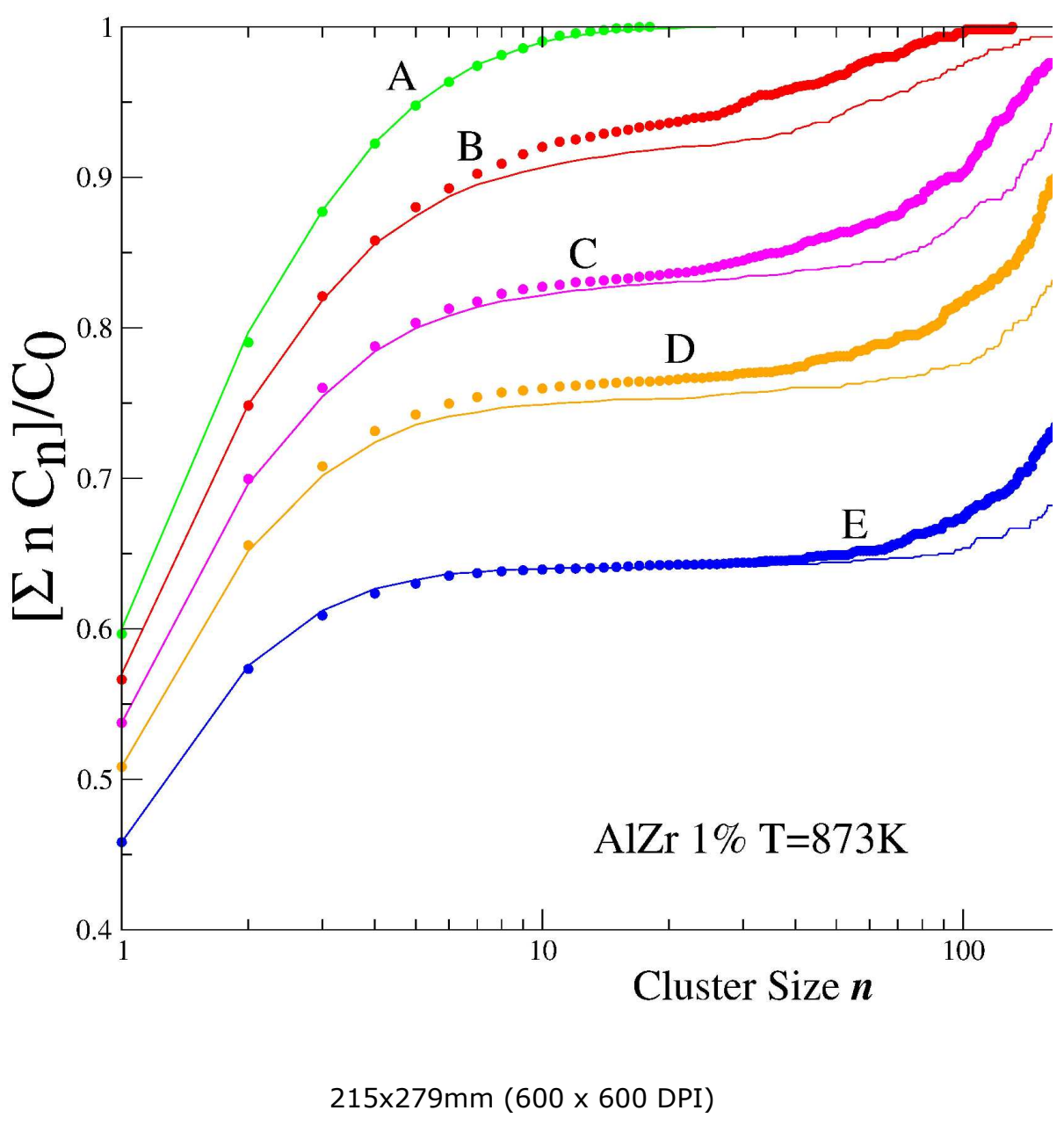

http://mc.manuscriptcentral.com/pm-pml 


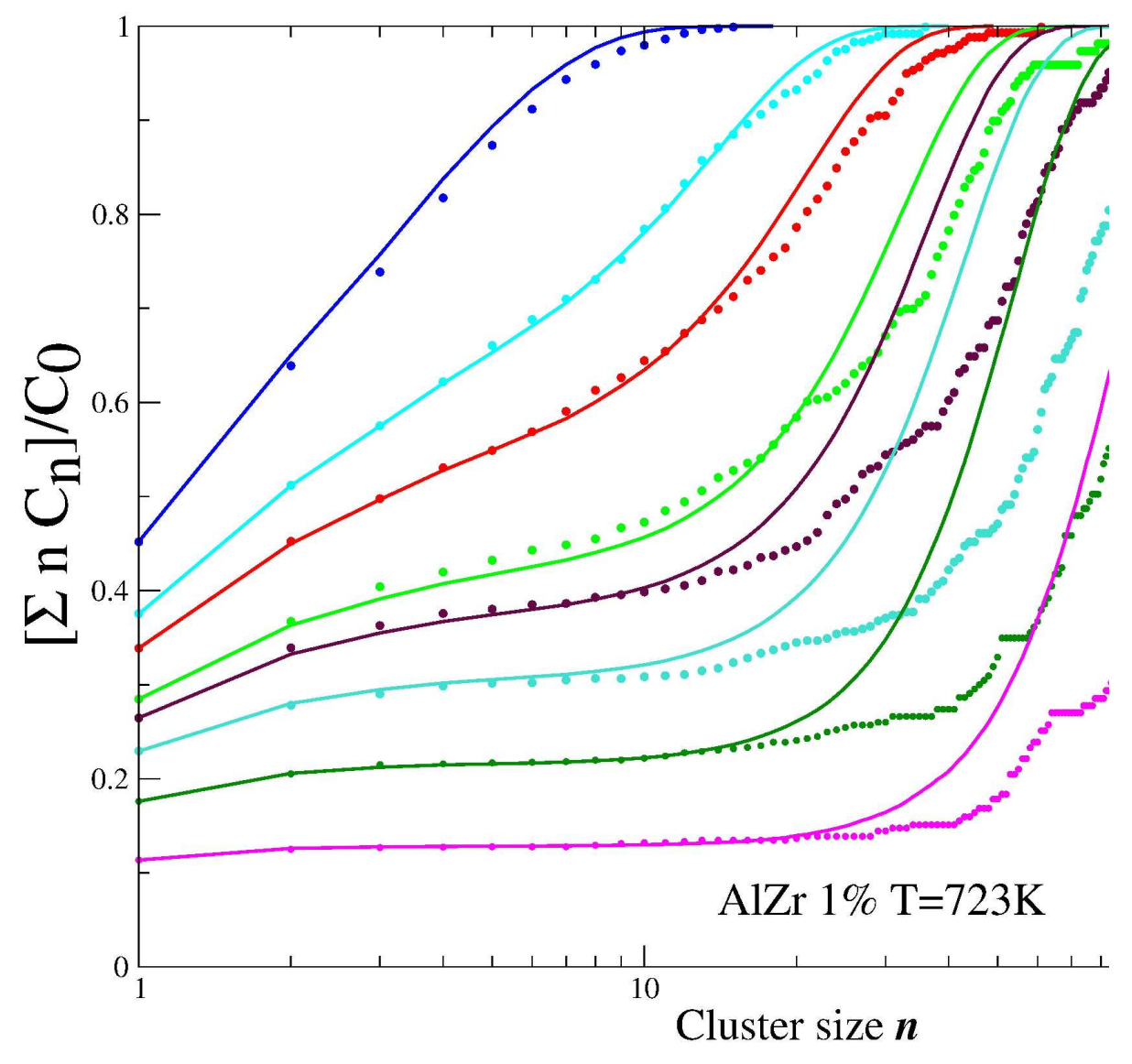

$215 \times 279 \mathrm{~mm}(600 \times 600 \mathrm{DPI})$

http://mc.manuscriptcentral.com/pm-pml 


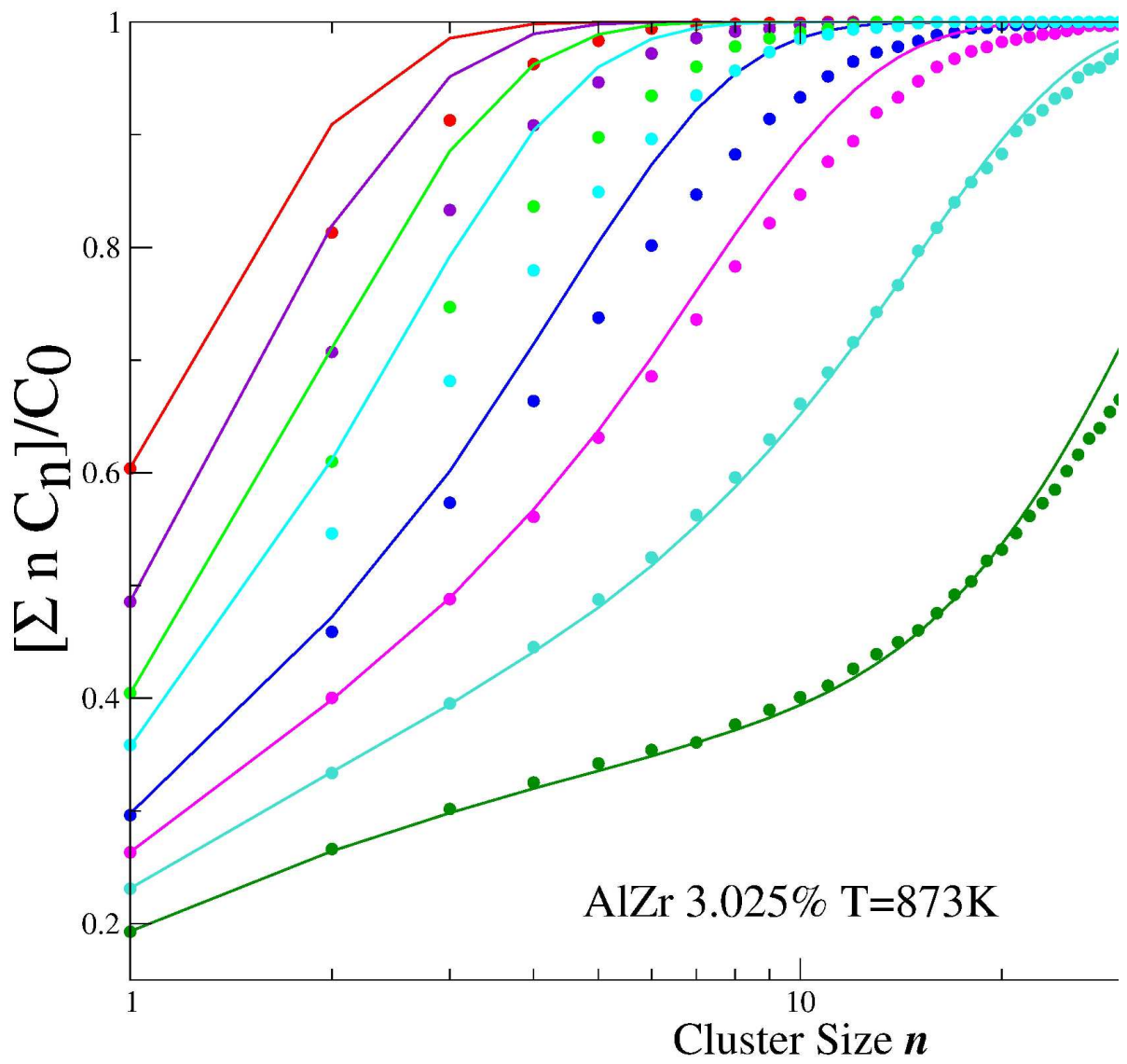

Figure 2: Comparison of KMC cumulated cluster distributions (symbols) with those expected at equilibrium (plain lines) for cases 1 (a), 2 (b) and 3 (c). In (a) letters A to E refer to particular distribution shown also in figures 1 and 3 . $215 \times 279 \mathrm{~mm}(600 \times 600 \mathrm{DPI})$ 


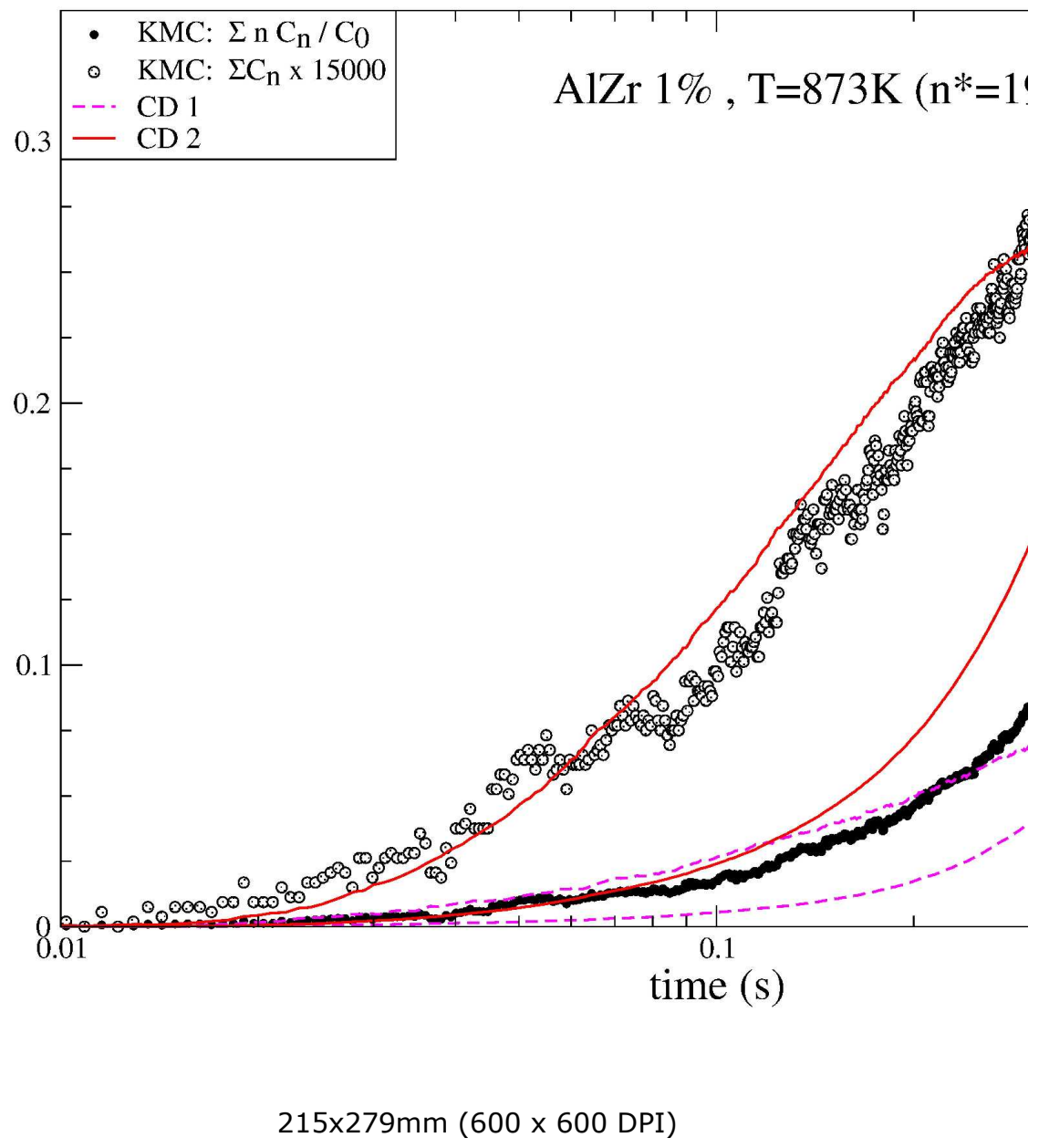

http://mc.manuscriptcentral.com/pm-pml 


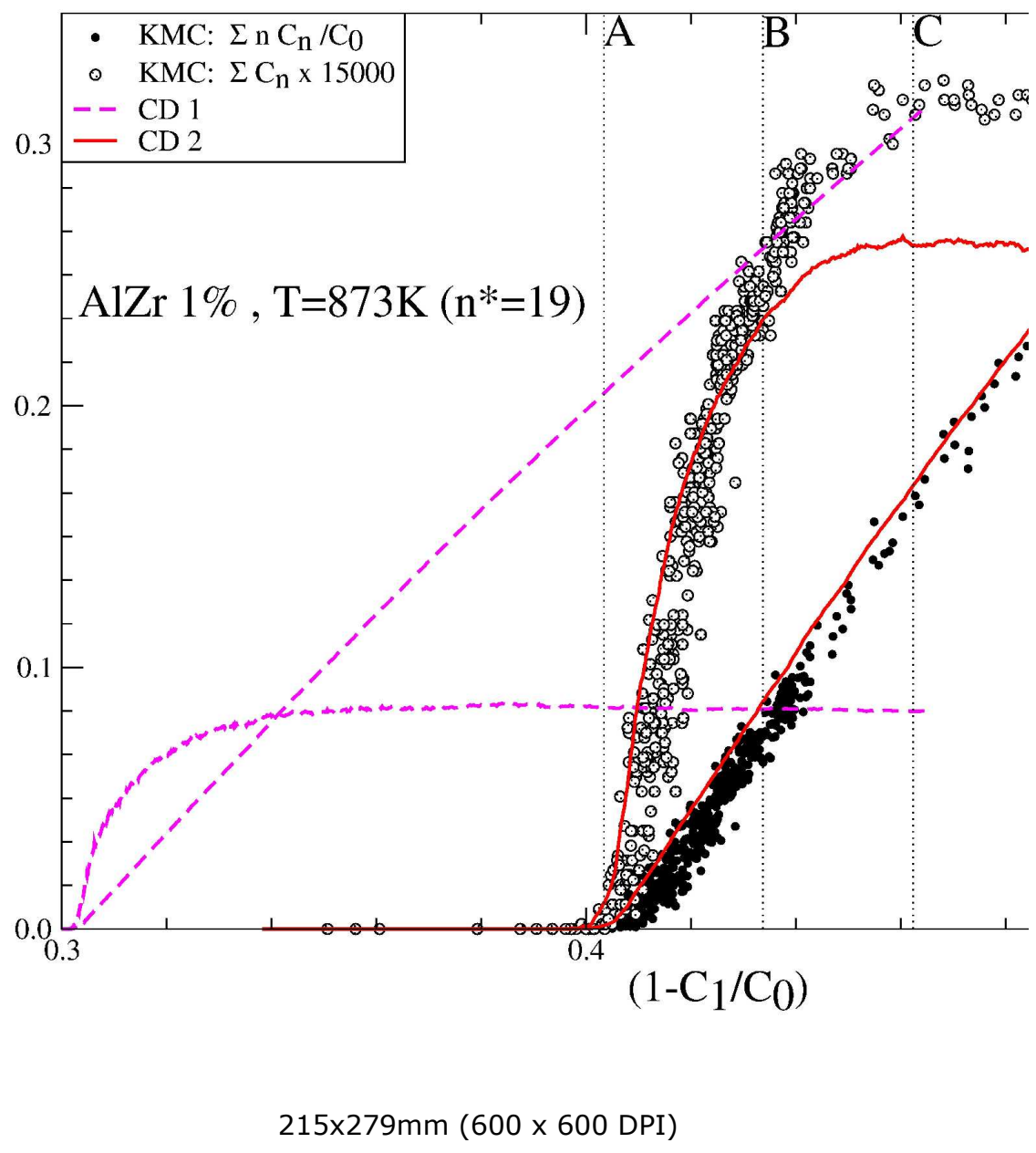

http://mc.manuscriptcentral.com/pm-pml 


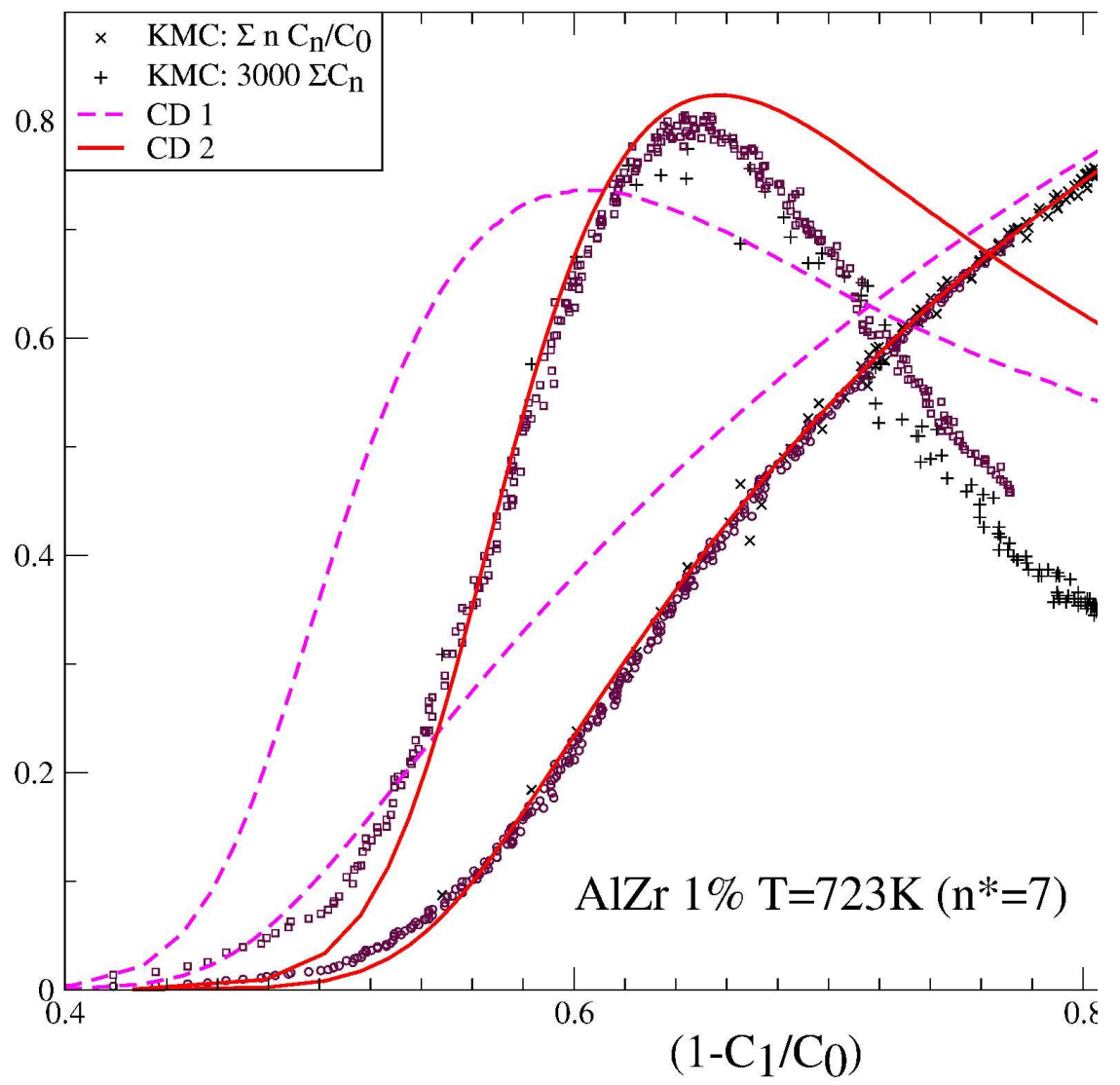

$215 \times 279 \mathrm{~mm}(600 \times 600 \mathrm{DPI})$ 


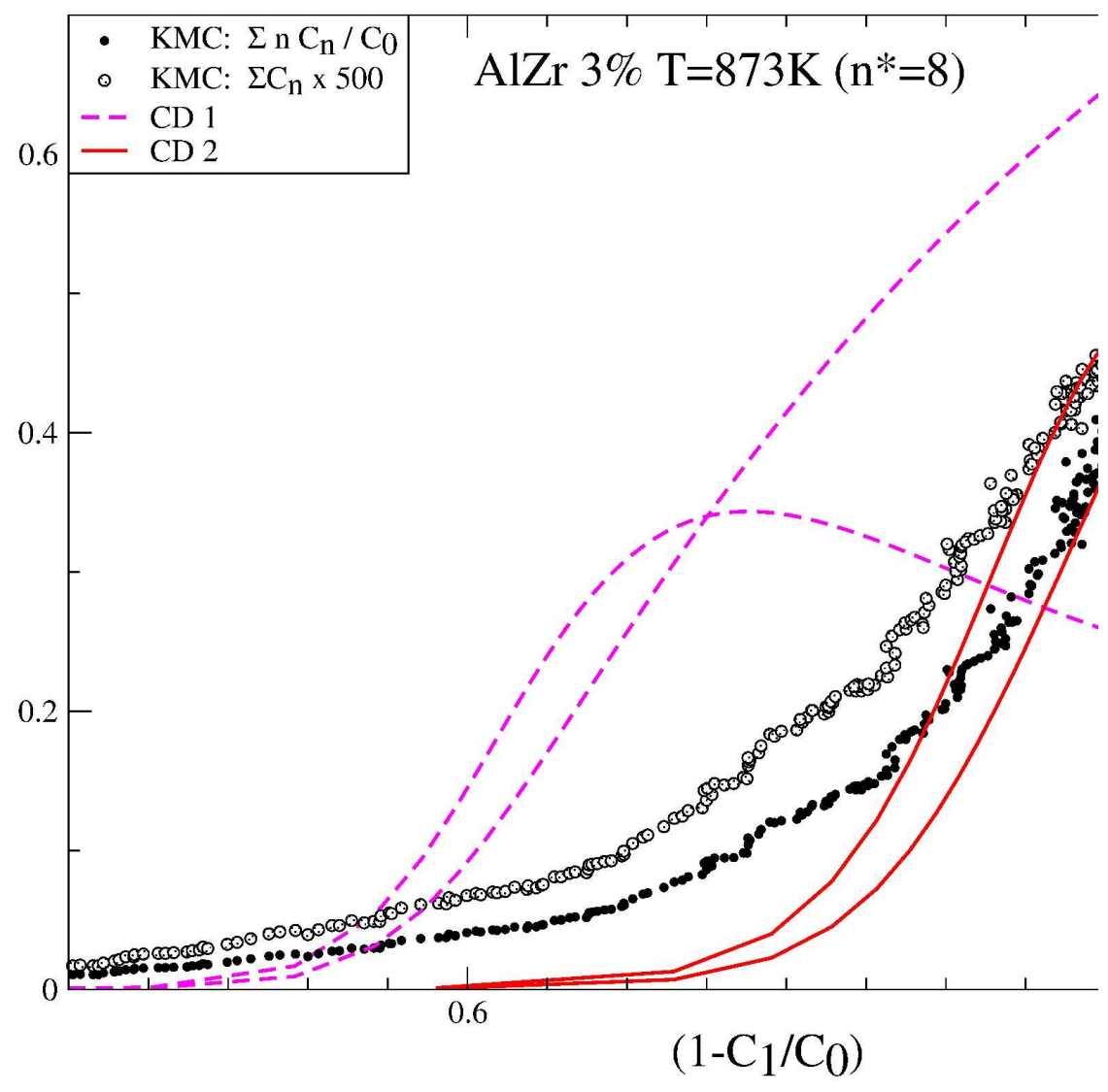

Figure 3: Comparison of two CD solutions with KMC simulations:

(a) and (b) AlZr $1 \%$ at $\mathrm{T}=873 \mathrm{~K}$ (case 1 )

(c) AlZr $1 \%$ at $\mathrm{T}=723 \mathrm{~K}$ (case 2 )

(d) AlZr 3\% at T=873K (case 3 )

The reported quantities are the precipitated fraction (plain symbols) and the number of precipitates (open symbols) multiplied by a convenient constant to fit the window. Results are reported vs. time

(a) or vs. (1- C1)/C0 (b, c and d) where $\mathrm{C} 1$ is the concentration in monomers and C0 the total solute concentration.

The two CD solutions are:

CD 1, with constant free surface energy, dashed line (magenta on line) $\mathrm{CD} 2$, the complete solution, plain line (red online)

Letters $A$ to $E$ in (b) refer to distributions shown in figure 2 (a) and figure 1. 


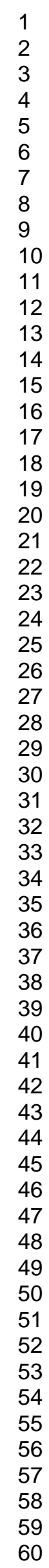

$215 \times 279 m m(600 \times 600 \mathrm{DPI})$

http://mc.manuscriptcentral.com/pm-pml 


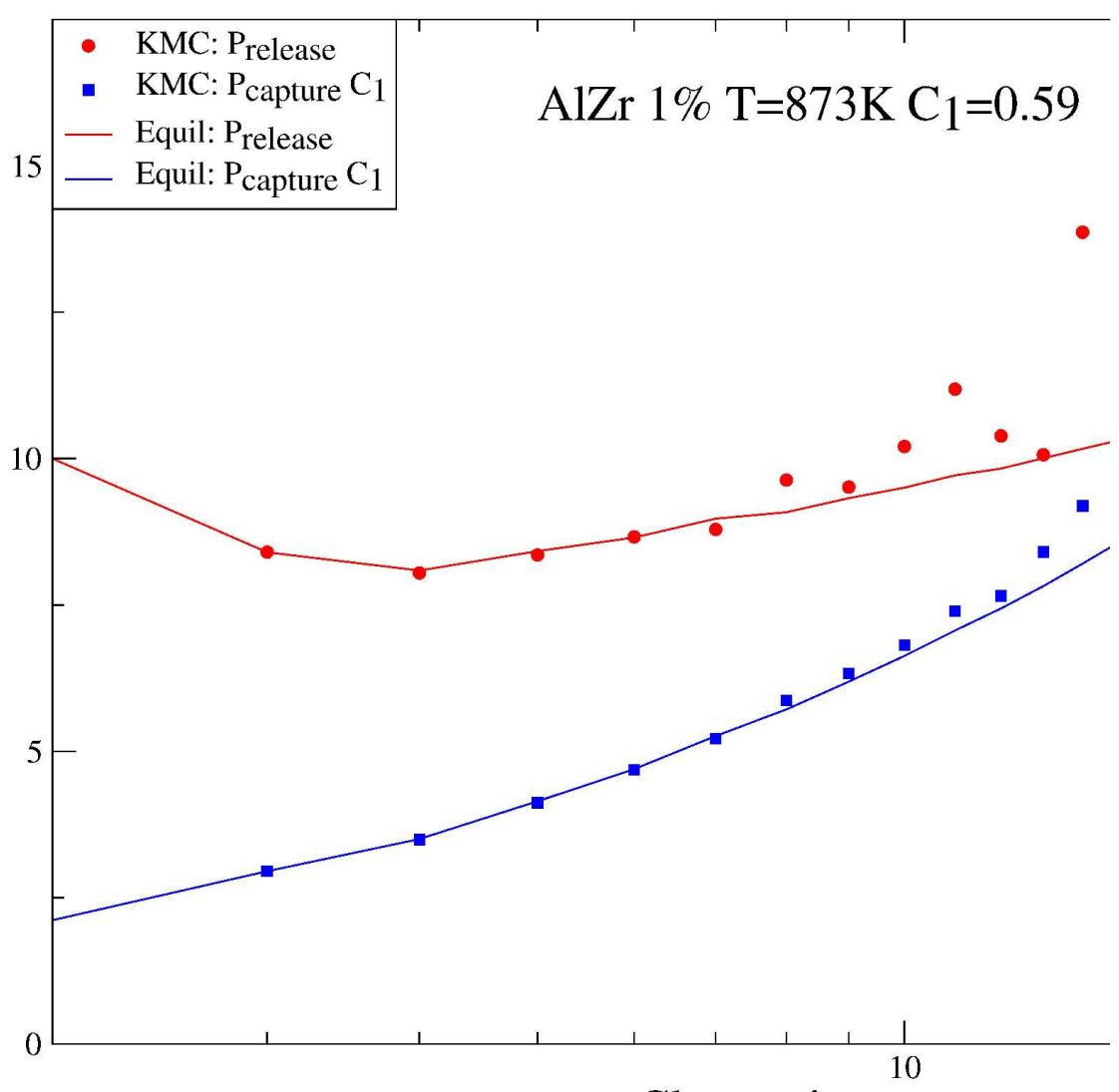

Cluster size $\boldsymbol{n}$

$215 \times 279 \mathrm{~mm}(600 \times 600 \mathrm{DPI})$ 


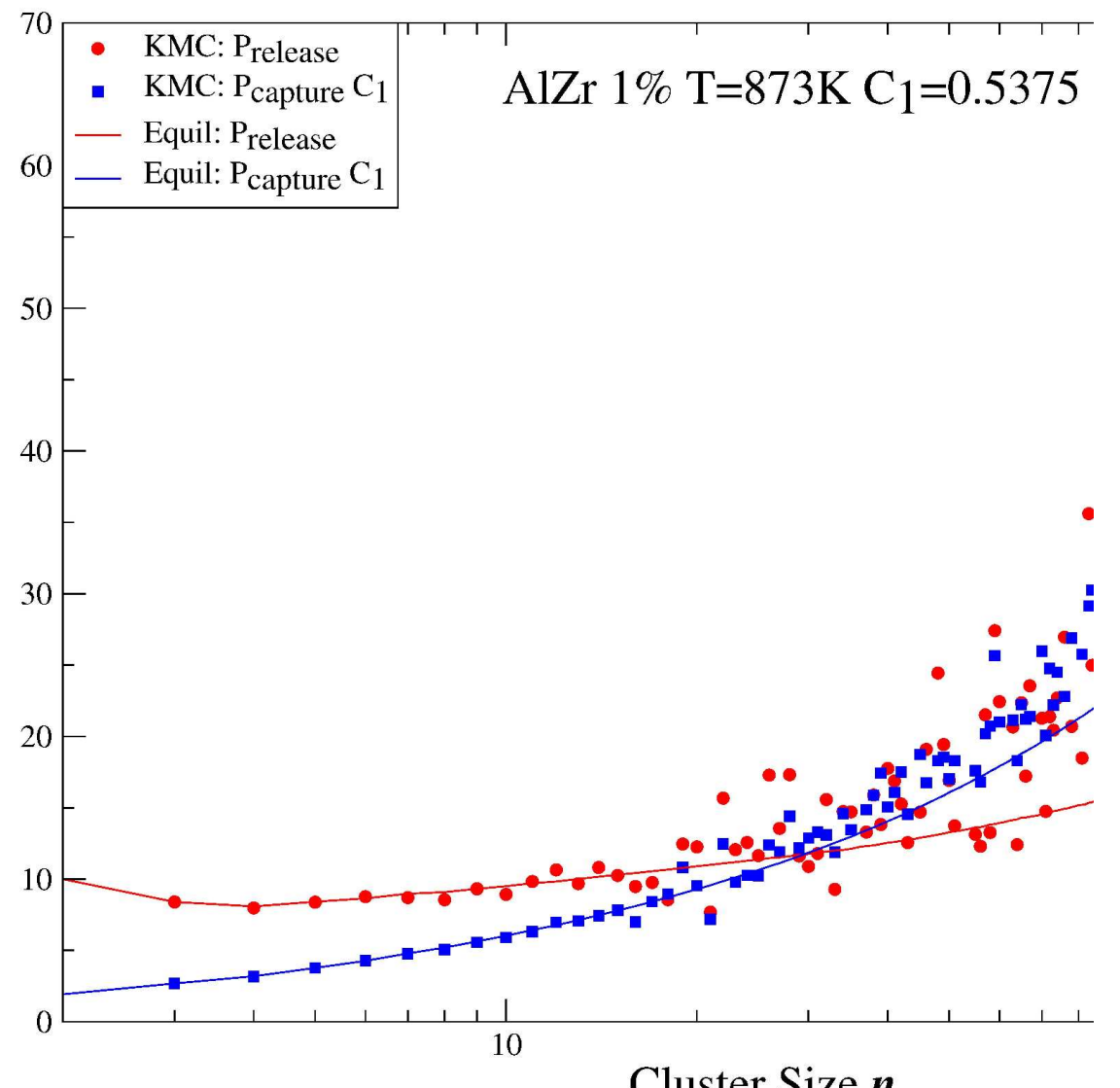

Figure 4: Typical behavior of the capture and release factors measured on KMC clusters for early (a) and late (b) stages of precipitation, compared with expected values at equilibrium (plain lines). The intersection of the two curves defines the instantaneous local critical cluster size $n^{*}$. NB: to simplify, both factors are normalized by $\exp (-\mathrm{Tw} / \mathrm{T})$ so that $\mathrm{P} 2,1=10$, an integer whose value can be found thanks to tables provided in Lepinoux (2005).

$$
215 \times 279 \mathrm{~mm}(600 \times 600 \mathrm{DPI})
$$




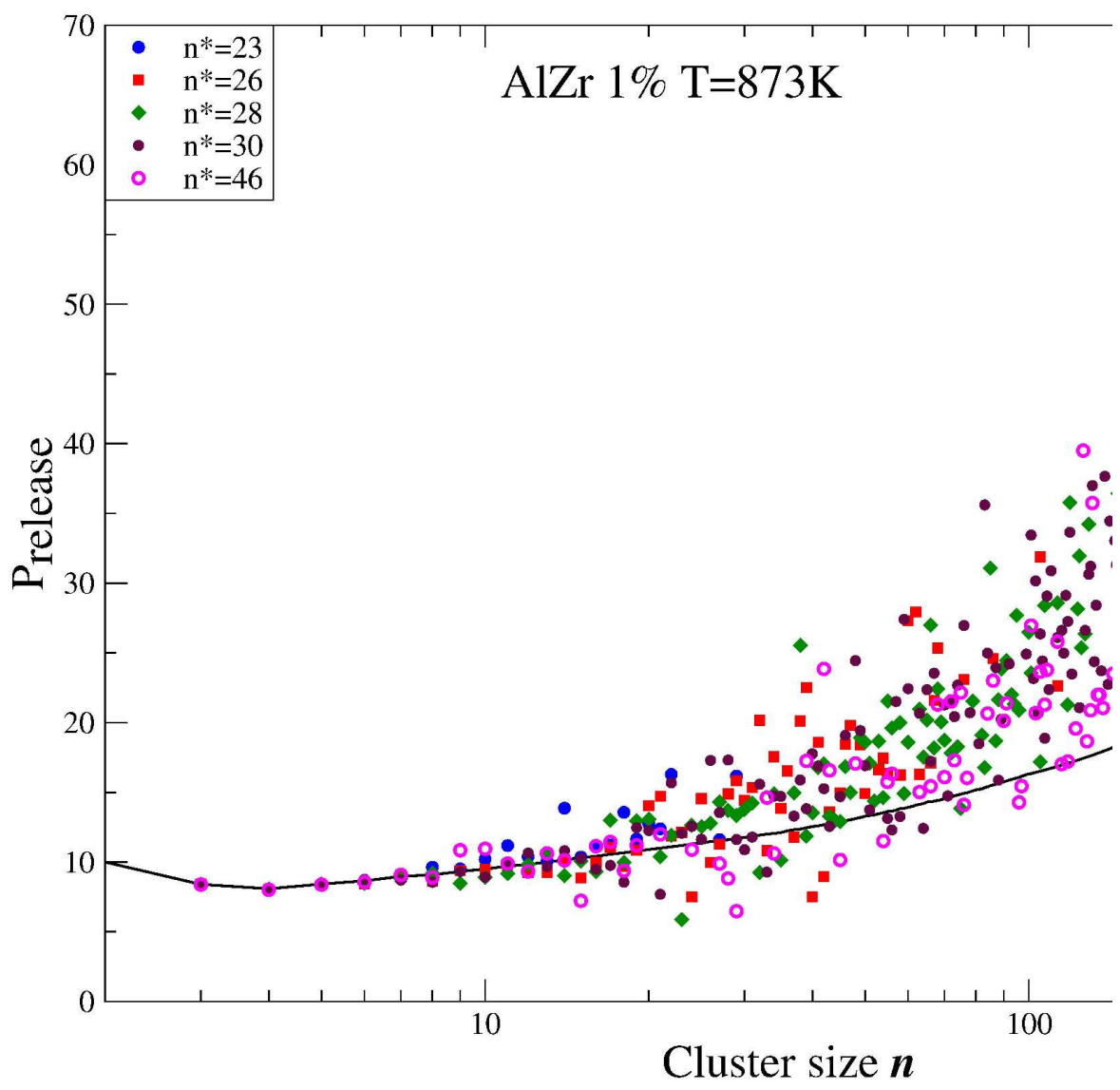

$215 \times 279 m m(600 \times 600$ DPI $)$ 


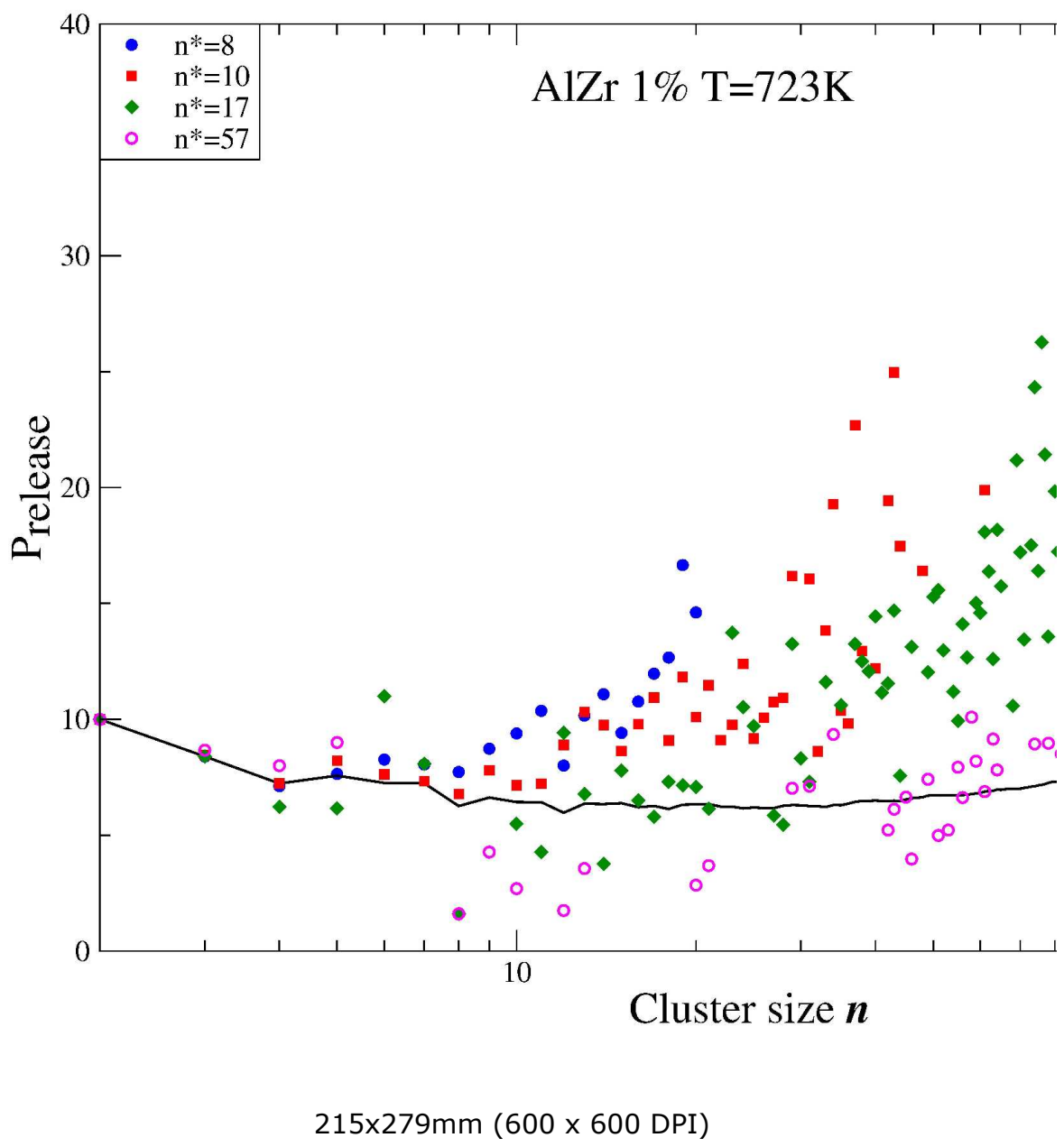

http://mc.manuscriptcentral.com/pm-pml 


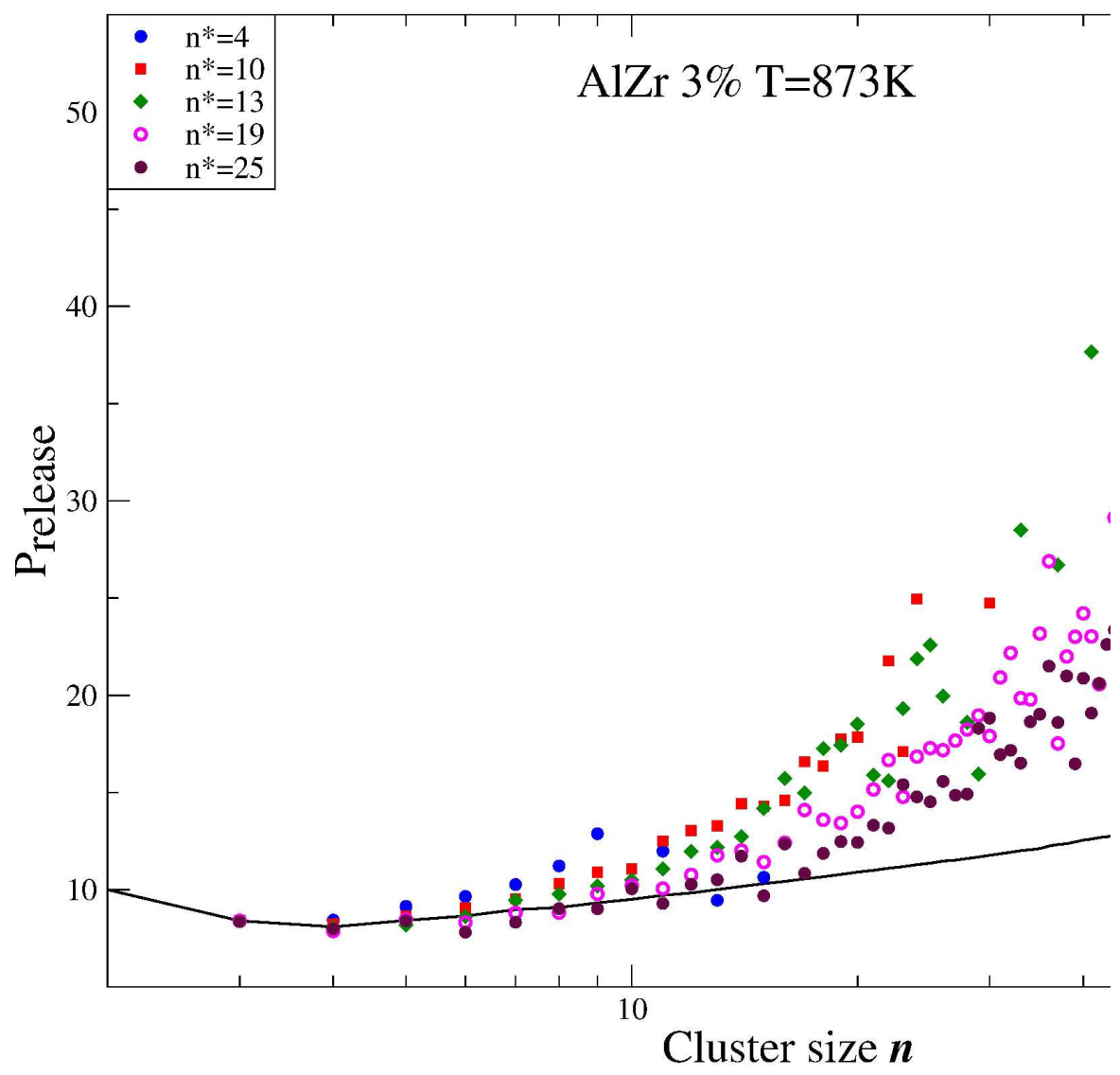

Figure 5: Evolution of the release factor for various values of the instantaneous local critical cluster size, for case 1 (a), case 2 (b) and case 3 (c). Symbols are related to KMC clusters and lines to expected values at thermodynamical equilibrium. As in figure 4 , the release factor is normalized by $\exp (-\mathrm{Tw} / \mathrm{T})$.

$215 \times 279 \mathrm{~mm}(600 \times 600 \mathrm{DPI})$ 


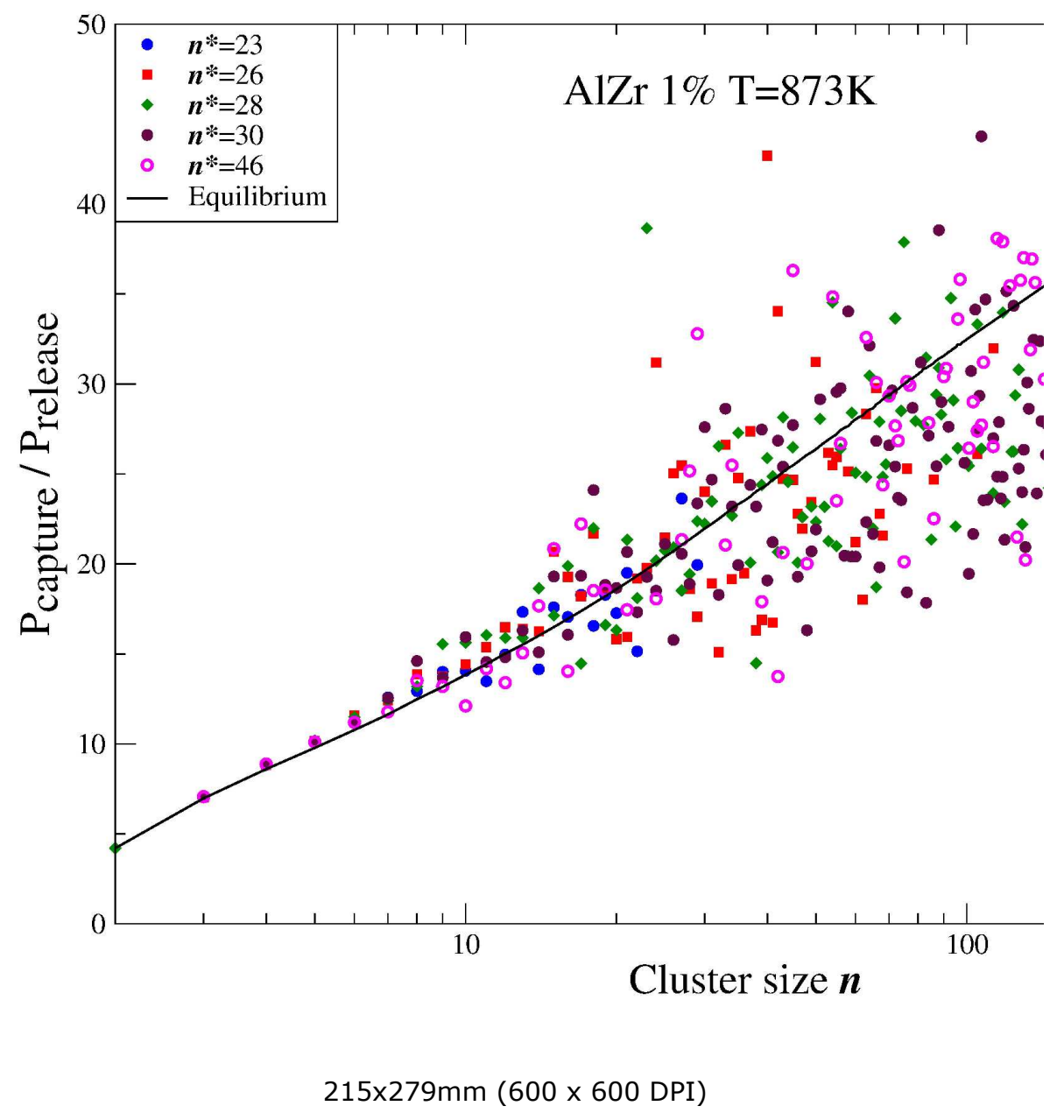

http://mc.manuscriptcentral.com/pm-pml 


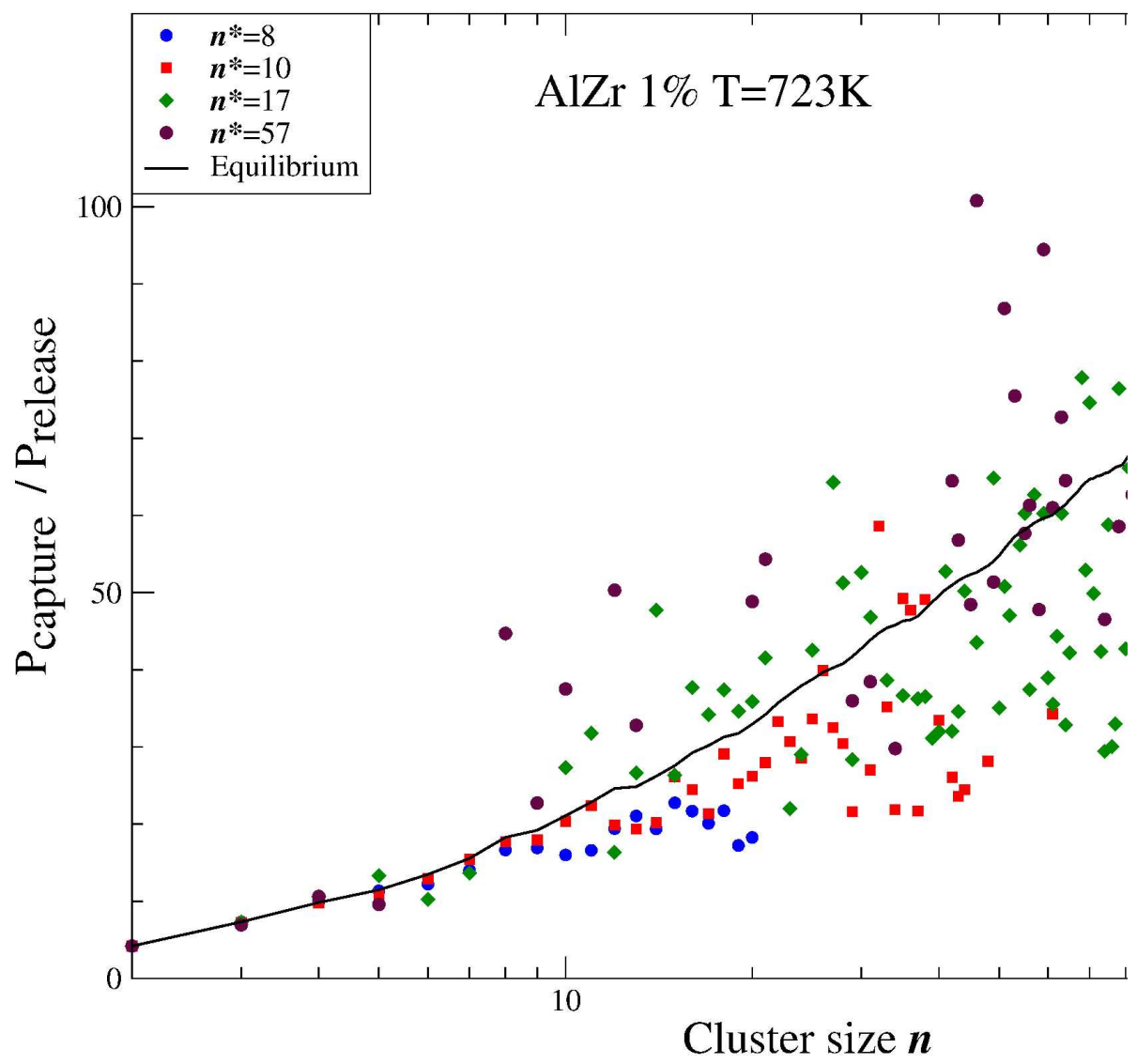

$215 \times 279 \mathrm{~mm}(600 \times 600 \mathrm{DPI})$ 


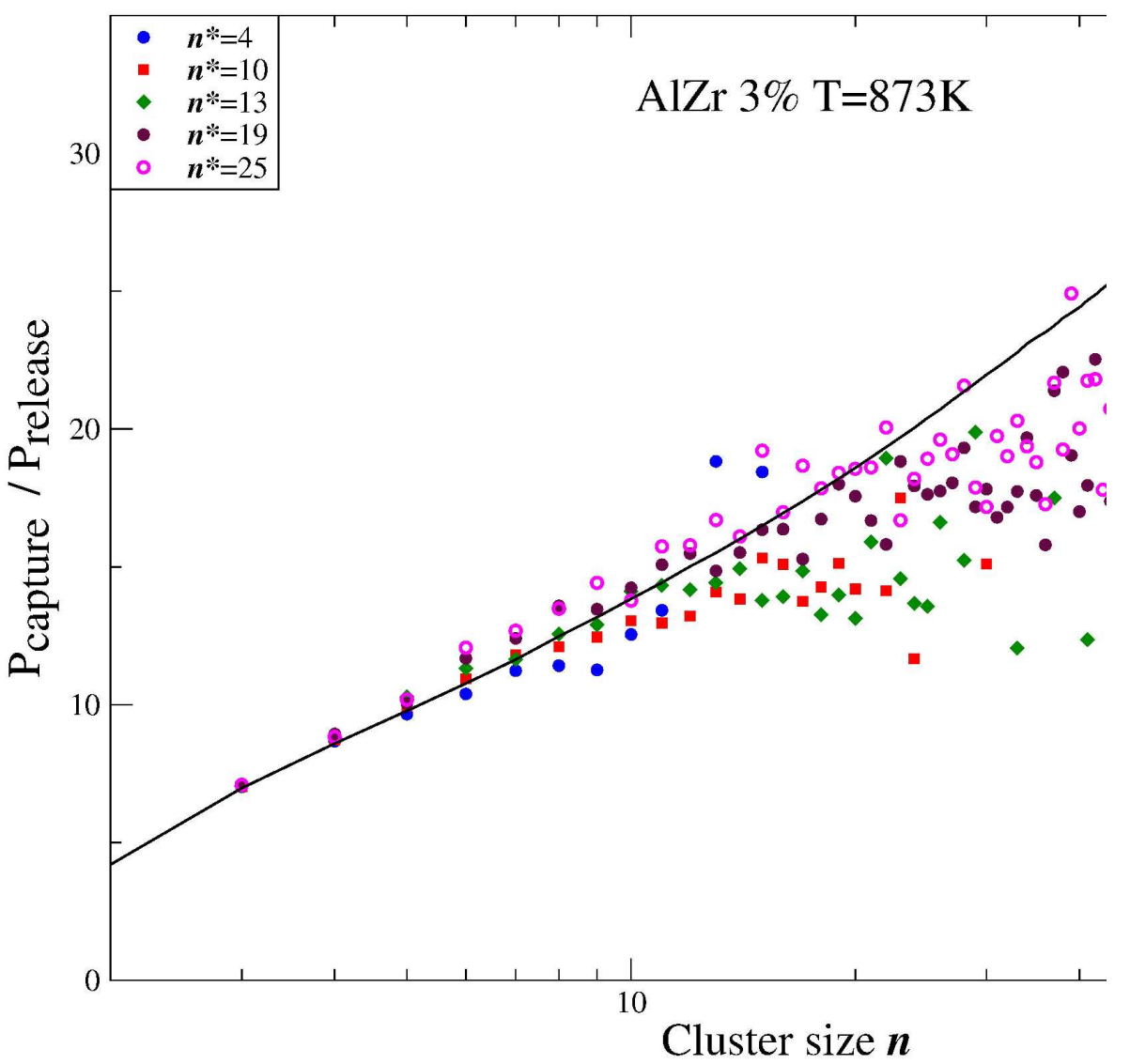

Figure 6: Evolution of the ratio of the capture and release factors for various values of the local critical cluster size, for case 1 (a), case 2 (b) and case 3 (c). Symbols are related to KMC clusters and lines to expected values at thermodynamical equilibrium.

NB: to simplify, the ratio is normalized by $\exp (-2 T w / T)$ so that the first value is equal to 4.2 whatever the temperature, a value which can be easily checked thanks to tables provided in Lepinoux (2005). $215 \times 279 \mathrm{~mm}(600 \times 600 \mathrm{DPI})$ 


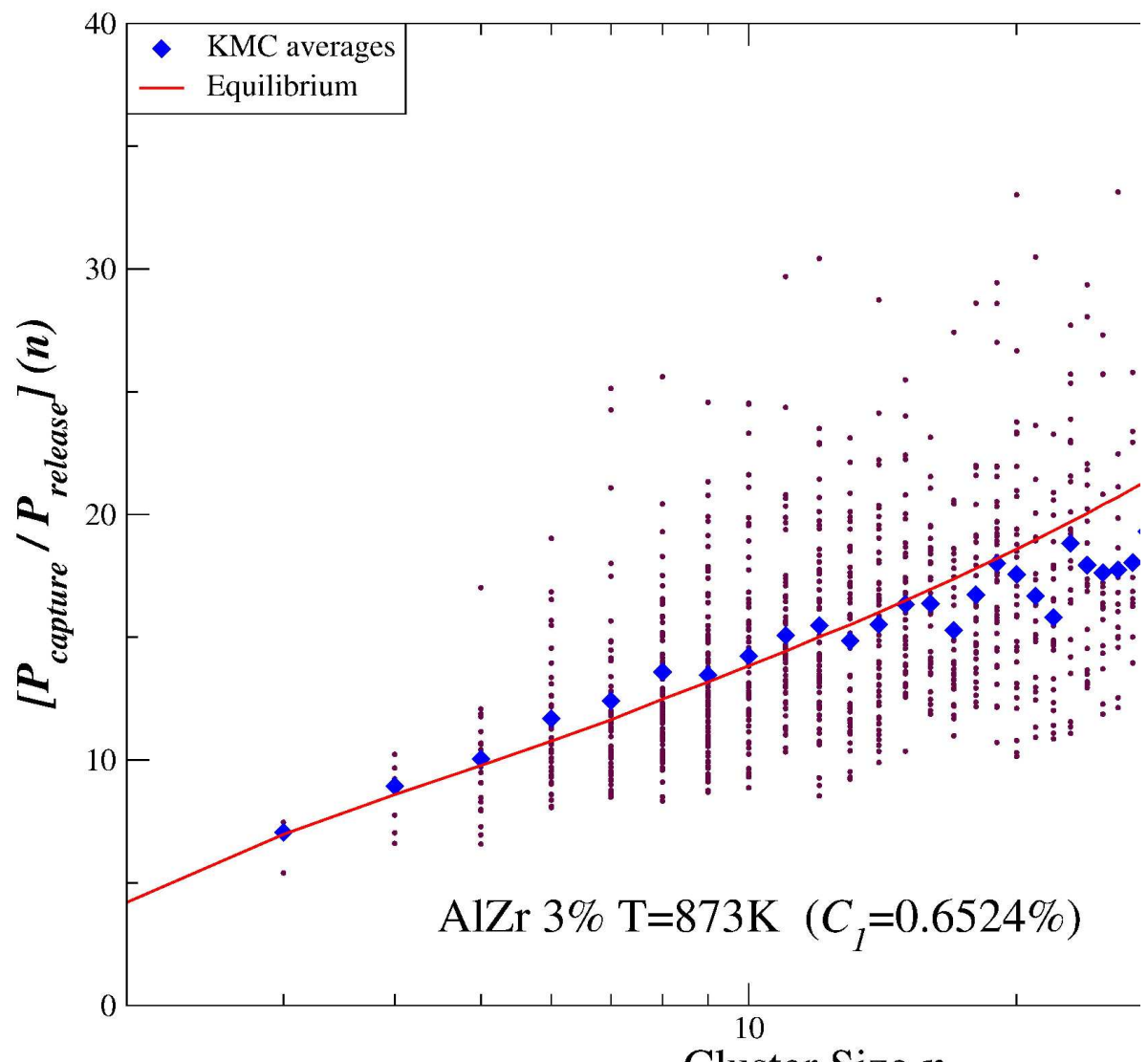

\section{Cluster Size $\boldsymbol{n}$}

Figure 7: The dispersion of cluster properties, here the ratio Pcapture / Prelease (normalized as in figure 6 ) as a function of the cluster size. Average values are represented by diamond symbols and individual values by small dots. The plain line represents the expected values at thermodynamical equilibrium $\left(n^{*}=19\right)$.

$215 \times 279 \mathrm{~mm}(600 \times 600 \mathrm{DPI})$ 


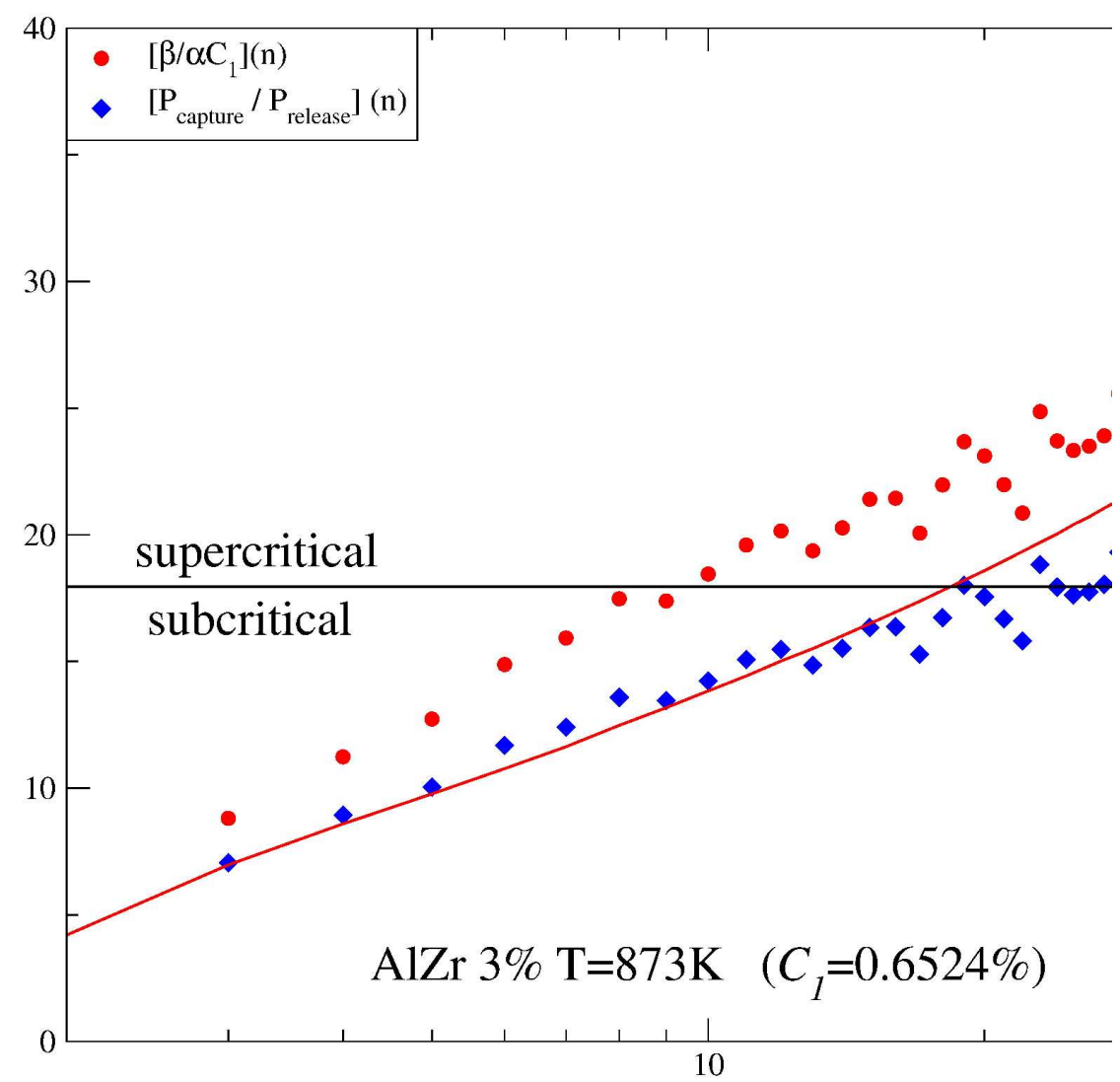

Cluster Size $\boldsymbol{n}$

Figure 8: Comparison of the ratios (Pcapture/Prelease) and $(\beta / \mathrm{aC} 1)$, both normalized by $\exp (-$ $2 \mathrm{Tw} / \mathrm{T}$ ) as in previous figures. For this alloy at $\mathrm{T}=873 \mathrm{~K}, \mathrm{Tw}=1.0721$. The height of the horizontal line defining the supercritical and subcritical ranges is: $1 /[\mathrm{C} 1 \exp (2 \mathrm{Tw} / \mathrm{T})]$. With present conditions its numerical values is equal to: 17.955 . $215 \times 279 \mathrm{~mm}(600 \times 600 \mathrm{DPI})$ 


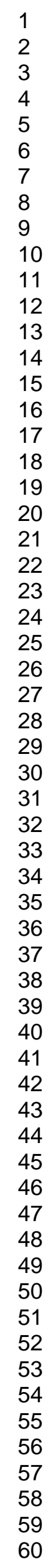

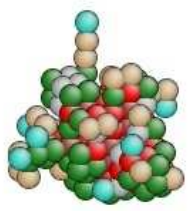

$323 \times 283 \mathrm{~mm}(72 \times 72 \mathrm{DPI})$

http://mc.manuscriptcentral.com/pm-pml 


1
2
3
4
5
6
7
8
9
10
11
12
13
14
15
16
17
18
19
20
21
22
23
24
25
26
27
28
29
30
31
32
33
34
35
36
37
38
39
40
41
42
43
44
45
46
47
48
49
50
51
52
53
54
55
56
57
59
60

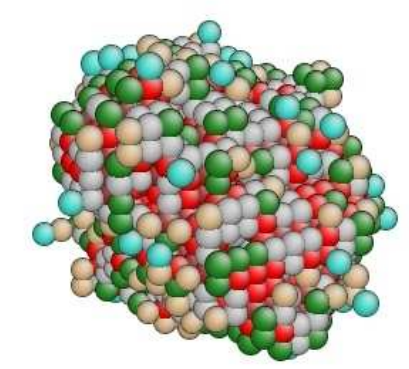

$323 \times 283 \mathrm{~mm}(72 \times 72 \mathrm{DPI})$

http://mc.manuscriptcentral.com/pm-pml 


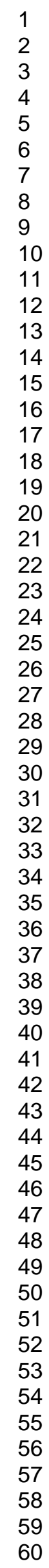

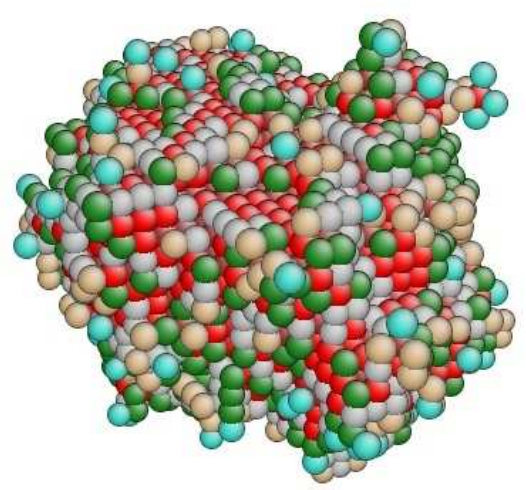

$323 \times 283 \mathrm{~mm}(72 \times 72 \mathrm{DPI})$

http://mc.manuscriptcentral.com/pm-pml 


1
2
3
4
5
6
7
8
9
10
11
12
13
14
15
16
17
18
19
20
21
22
23
24
25
26
27
28
29
30
31
32
33
34
35
36
37
38
39
40
41
42
43
44
45
46
47
48
49
50
51
52
53
54
55
56
57
58
59
60

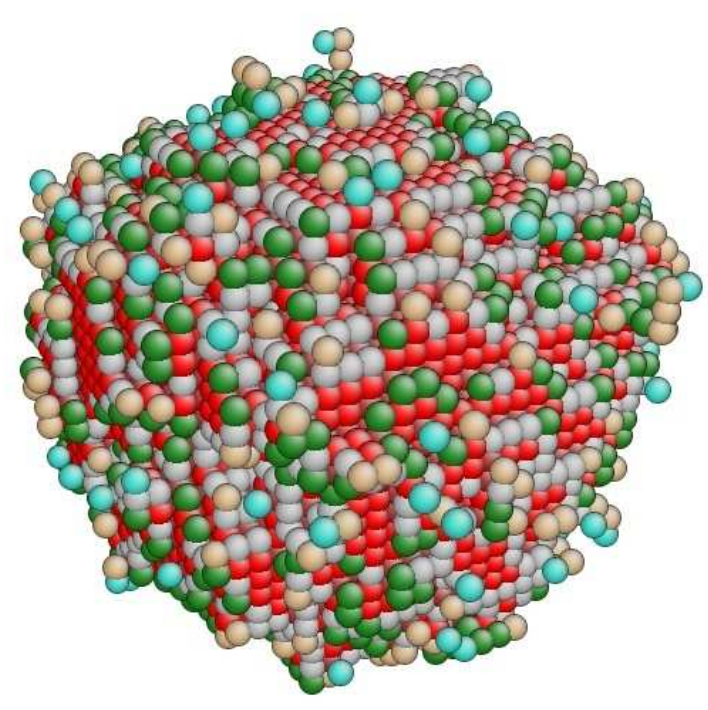

$323 \times 283 \mathrm{~mm}(72 \times 72 \mathrm{DPI})$

http://mc.manuscriptcentral.com/pm-pml 
1

2

3

4

5

6

7

8

9

10

11

12

13

14

15

16

17

18

19

20

21

22

23

24

25

26

27

28

29

30

31

32

33

34

35

36

37

38

39

40

41

42

43

44

45

46

47

48

49

50

51

52

53

54

55

56

57

58

59

60
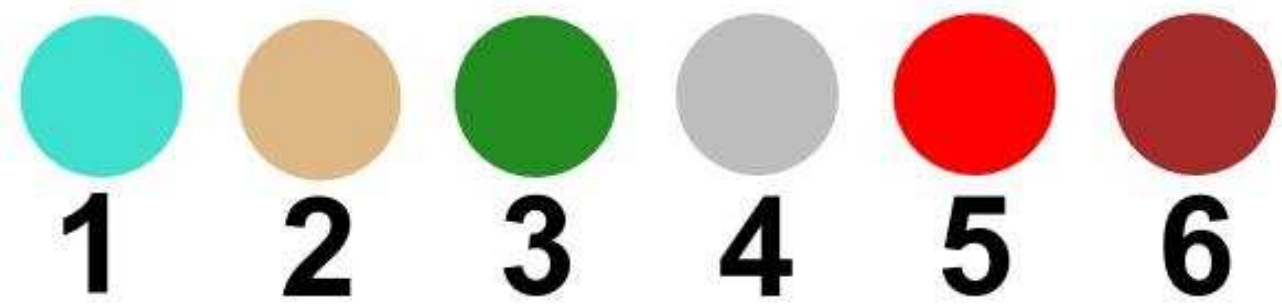

Figure 9: Typical growth of an isolated precipitate from a germ containing about 100 solute atoms (a) up to its final size about 15000 solute atoms (d), in equilibrium with the solid solution. The solute concentration, a few 10-3, was choosen high enough to trigger precipitation in a reasonable amount of computation time and small enough to select only one precipitate in the KMC simulation box (106 atomic sites) at $\mathrm{T}=873 \mathrm{~K}$. The macroscopic shape is roughly cubic with $\{100\}$ faces.

Although at such low solute concentration the precipitate remains fairly compact, growth is not monotonous and important changes of shape due to the capture of nearby fluctuations of various size can be observed. (e) color chart for solute atoms as a function of the number of links with other solute atoms. 


1
2
3
4
5
6
7
8
9
10
11
12
13
14
15
16
17
18
19
20
21
22
23
24
25
26
27
28
29
30
31
32
33
34
35
36
37
38
39
40
41
42
43
44
45
46
47
48
49
50
51
52
53
54
55
56
57
59
60
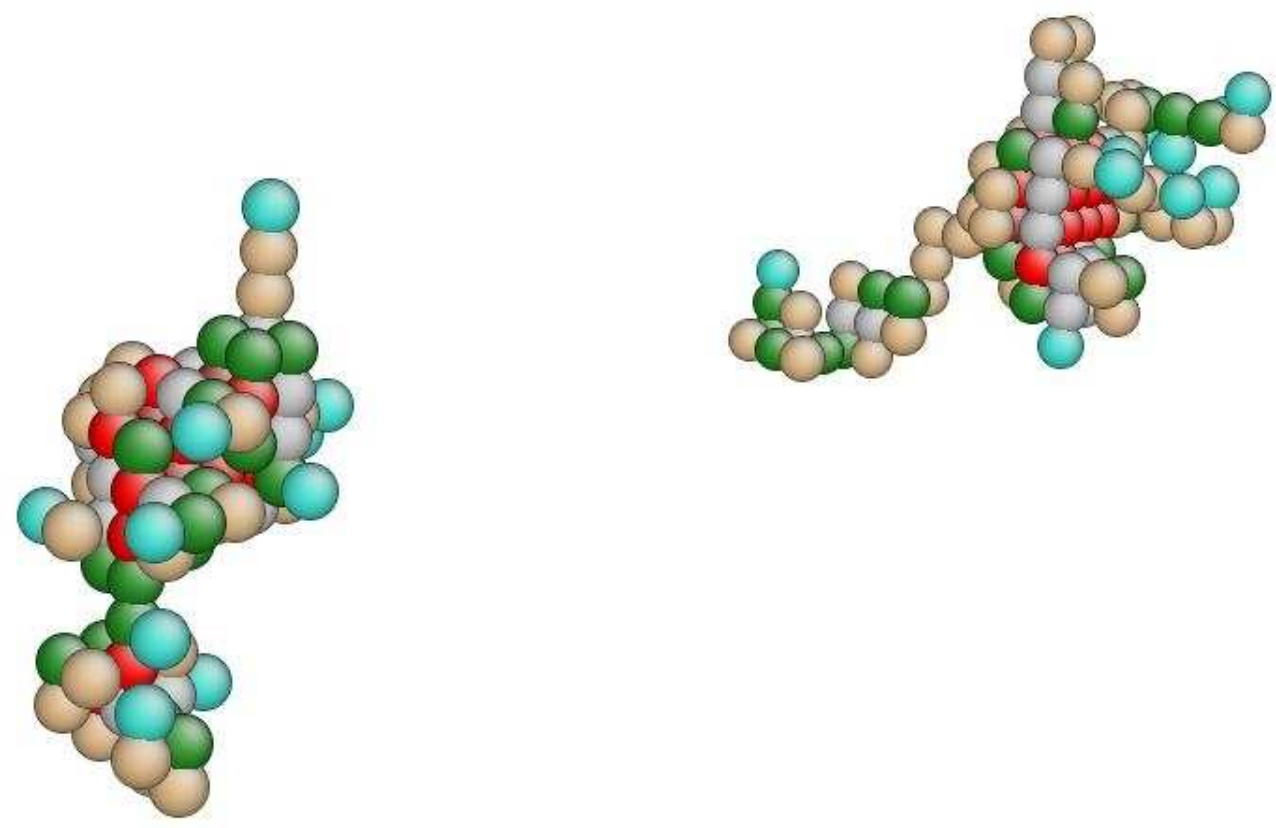

30 

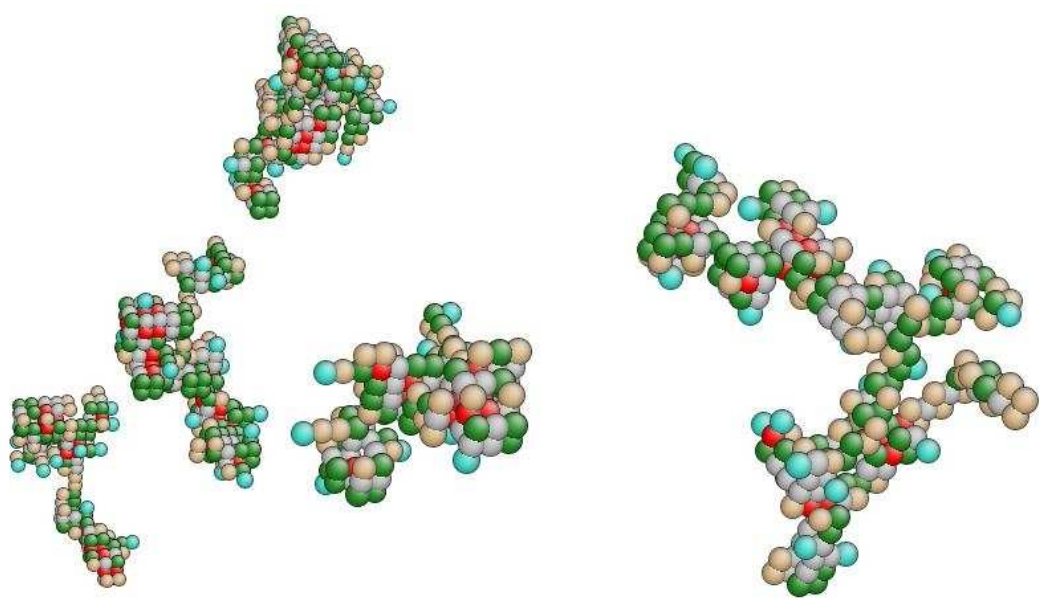

Figure 10: Typial shapes of clusters observed at solute concentration of $1 \%$ (a) to $5 \%$ (b), $T=873 \mathrm{~K}$. Clusters are increasingly composed of aggregates of smaller clusters loosely interconnected. Although the size of clusters increase, the largest ones are not necessarily the most stable ones (cf. figure 9 for the color chart). 


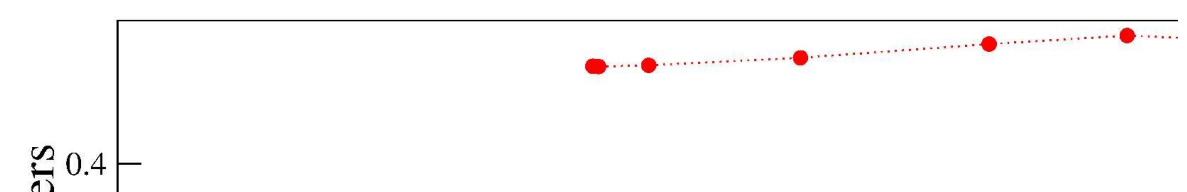

AlZr 3\% 873K

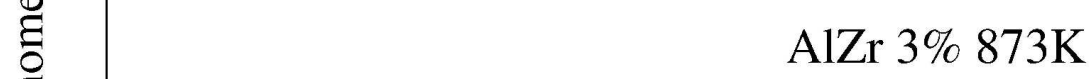

Figure 11: Evolution of the fraction of atomic sites where a monomer cannot be located without bonding with another solute atom, as a function of $(1-\mathrm{C} 1) / \mathrm{C} 0$ where $\mathrm{C} 1$ is the concentration in monomers and $\mathrm{CO}$ the total solute concentration. For $\mathrm{C} 0=1 \%$ the first stage is a plateau, while for $\mathrm{CO}=3 \%$ a bump appears before a fast decreasing.

$215 \times 279 \mathrm{~mm}(600 \times 600 \mathrm{DPI})$ 